\title{
From Liquid- to Solid-State Batteries: Ion Transfer Kinetics of Heteroionic Interfaces
}

\author{
Manuel Weiss ${ }^{1,2} \cdot$ Fabian J. Simon $^{1,2} \cdot$ Martin R. Busche ${ }^{1,2} \cdot$ Takashi Nakamura $^{3} \cdot$ Daniel Schröder $^{1,2} \odot$. \\ Felix H. Richter ${ }^{1,2}$. Jürgen Janek ${ }^{1,2}$ (1)
}

Received: 9 August 2019 / Revised: 6 December 2019 / Accepted: 8 January 2020 / Published online: 18 March 2020

(c) The Author(s) 2020

\begin{abstract}
Hybrid battery cells combining liquid electrolytes (LEs) with inorganic solid electrolyte (SE) separators or different SEs and polymer electrolytes (PEs), respectively, are developed to solve the issues of single-electrolyte cells. Among the issues that can be solved are detrimental shuttle effects, decomposition reactions between the electrolyte and the electrodes, and dendrite propagation. However, the introduction of new interfaces by contacting different ionic conductors leads to other problems, which cannot be neglected before commercialization is possible. The interfaces between the different types of ionic conductors (LE/SE and PE/SE) often result in significant charge-transfer resistances, which increase the internal resistance considerably. This review highlights studies evaluating the interfacial resistances and activation barriers in such systems to present an overview of the issues still hampering hybrid battery systems. The interfaces between different SEs in hybrid all-solid-state batteries (SSBs) are considered as well. In addition, a short summary of physicochemical models describing heteroionic interfaces-interfaces between two different ion conductors-is given in an attempt to explain high interface resistances. In doing so, we hope to inspire future work on the crucial topic of interface optimization toward better SSBs.
\end{abstract}

Keywords Post LIB $\cdot$ Electrolyte $\cdot$ EIS $\cdot$ Polymer $\cdot$ Interphase $\cdot$ Protective layer

\section{Introduction}

Lithium-ion batteries (LIBs) have become the most widely applied battery technology since their commercialization in the 1990s [1, 2]. Besides their use in portable electronic devices, their application in electric vehicles gains importance rapidly. However, for electric vehicles to fully replace combustion-powered vehicles, higher energy and power

Electronic supplementary material The online version of this article (https://doi.org/10.1007/s41918-020-00062-7) contains supplementary material, which is available to authorized users.

Jürgen Janek

juergen.janek@pc.jlug.de

1 Institute of Physical Chemistry, Justus Liebig University Giessen, Heinrich-Buff-Ring 17, 35392 Giessen, Germany

2 Center for Materials Research (LaMa), Justus Liebig University Giessen, Heinrich-Buff-Ring 16, 35392 Giessen, Germany

3 Institute of Multidisciplinary Research for Advanced Materials, Tohoku University, 2-1-1, Katahira, Aoba-ku, Sendai 980-8577, Japan densities than what can be achieved using the current generation of LIB systems are needed.

The liquid electrolytes (LEs) currently used in many LIBs exhibit high ionic conductivities and enable fast interface kinetics because of excellent wetting of the porous electrodes [3]. They are not without disadvantages, though: LEs degrade easily thermally and electrochemically and suffer from concentration polarization because the LE is not solely limited to $\mathrm{Li}^{+}$transport $[4,5]$. In addition, LEs pose safety risks because of their high flammability. Furthermore, batteries with LEs require porous separators and are prone to leaking and gassing.

With solid electrolytes (SEs), the mechanical stability of the cell may be improved while enabling simplified cell assembly. Leakage is not an issue in all-solid-state batteries (SSBs) [4], which exclusively consist of solid components. Hence, SSBs are of major interest to possibly replace LIBs in the future [6-8] and are mostly based on inorganic SEs like alkali-ion-conducting oxides or thiophosphates. There are also serious attempts to construct SSBs using polymer electrolytes (PEs), that is, a conducting salt dissolved in a polymer matrix with sufficient structural 
rigidity at room-temperature to be regarded as solid [9]. To date, PEs show lower conductivities than good inorganic SEs, and PE-based SSBs are usually operated at elevated temperature.

For the following discussion of interfaces, it is important to understand the major differences between LEs/PEs and SEs. LEs and PEs show extremely low partial electronic conductivity and, therefore, can be considered as pure ionic conductors. In contrast, mobile electrons actually exist in SEs, although the electronic conductivity is small in most cases. Therefore, local equilibrium of ions, electrons, and the neutral component Li can be assumed in SEs. For thermodynamics and defect chemistry of lithium-ion conductors the reader is referred to respective literature [10].

Neither batteries only relying on LEs, nor SSBs created a single solution for reaching the theoretical energy and power density so far. Hence, hybrid systems are increasingly considered as potential solutions to the problems existent in single-electrolyte batteries. Different hybrid cell setups are depicted in Fig. 1.

One typical hybrid concept is using SEs as ion-selective membranes in batteries based on LEs as shown in Fig. 1a. It is employed in next-generation batteries like the $\mathrm{Li}-\mathrm{O}_{2}$ and the $\mathrm{Li}-\mathrm{S}$ systems, which are hampered by detrimental chemical cross-talk between the electrodes-shuttling of redox mediators in redox catalyzed $\mathrm{Li}-\mathrm{O}_{2}$ batteries $[11,12]$ and the polysulfide shuttle in $\mathrm{Li}-\mathrm{S}$ batteries, respectively [13-16]. The application of SE membranes was reported to improve redox-mediated $\mathrm{Li}-\mathrm{O}_{2}$ batteries [17-19] and to mitigate polysulfide shuttling in $\mathrm{Li}-\mathrm{S}$ batteries [20-25]. Additionally, SE separators can prevent dendrites from reaching the cathode, mitigating the risk of short circuits $[7,26]$.

Sandwich or bilayer setups (Fig. 1b) can be used either for the combination of a PE with an SE [22, 23, 27-29] or for two different SEs [30]. PEs can be stabler against $\mathrm{Li}$ metal [7], thus preventing SE degradation at the anode, and are able to retain contact despite occurring volume changes. Further, they may suppress dendrite nucleation because of the homogeneous $\mathrm{Li}^{+}$ion flux at the interface [8, 27]. A stable interface without dendrite propagation was predicted in case a sufficiently high shear modulus is reached [31, 32]. Recent calculations proposed the inhibition of dendrite formation for a shear modulus ten times higher than that of lithium metal [33]. In addition, dendrite formation can also be prevented by increased yield strength of the PE or reduced current density [33]. Sandwich-type SSBs using two different SEs are constructed to achieve good compatibility with both electrodes. Ideally, one electrolyte is stable against reduction in contact with the anode and the other one is stable against oxidation in contact with the cathode [30]. This concept is successfully used in solid oxide fuel cells, where $\mathrm{CeO}_{2}$ is protected against reduction by yttria-stabilized zirconia (YSZ) [34].
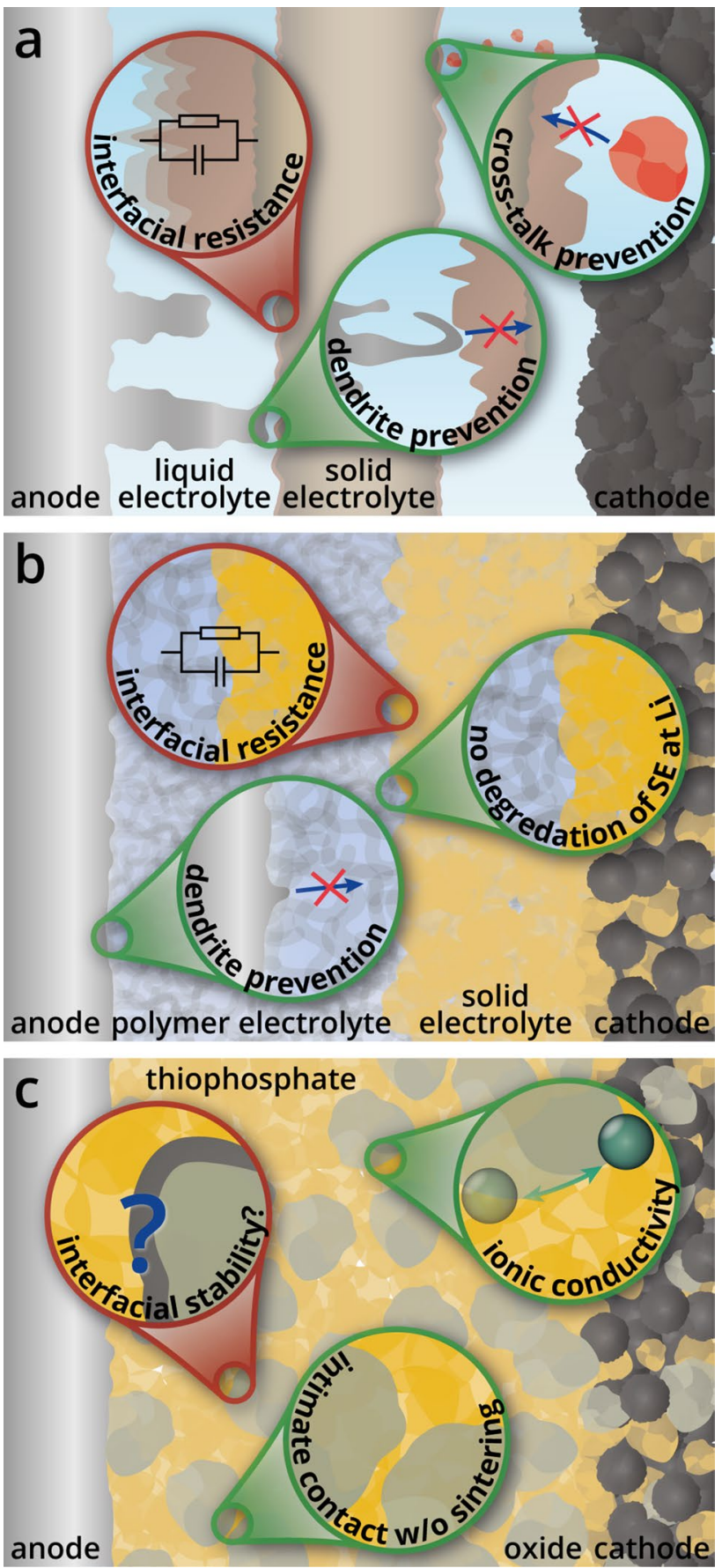

Fig. 1 Possible architectures of hybrid battery systems: a a solid separator in a cell working with liquid electrolyte (LE). Depicted here is a non-aqueous $\mathrm{Li}-\mathrm{O}_{2}$ cell with a porous carbon cathode and a $\mathrm{Li}$ anode. The same setup applies for $\mathrm{Li}-\mathrm{S}$ cells with sulfur or carbon cathodes. $\mathbf{b}$ a bilayer hybrid cell setup consisting of the polymer electrolyte (PE) and the SE with a cathode composite comprised of a transition metal oxide and an SE. In addition, two different SEs can also be combined in a sandwich setup. c distribution of SE particles in a matrix consisting of another type of the SE. PE matrices with ceramic fillers can also be realized in this way. All types of hybrid cells try to solve the problems of single-electrolyte approaches by preventing dendrites or cross-talk and the contact between Li and the thiophosphate SE, which leads to degradation. In addition, enhanced ionic conductivity and high densities close to theoretical density at lower processing temperatures can be achieved. However, these advantages are often accompanied by significant interface resistances and poor interfacial stability with possible interphase formation 
Particles of one ionic conductor immersed in a host matrix of a different electrolyte are the third concept, which is depicted in Fig. 1c. For the combination of an oxide $\mathrm{SE}$ with a thiophosphate one- $\mathrm{Li}_{7} \mathrm{La}_{3} \mathrm{Zr}_{2} \mathrm{O}_{12}$ (LLZO) and $\beta-\mathrm{Li}_{3} \mathrm{PS}_{4}$ (LPS) for example - the processing temperature can be reduced compared to the pure oxide and the ionic conductivity is enhanced because of space-charge effects at the interface [35, 36]. SE fillers are also commonly used in polymer matrices [37-40]. The ionic conductivity and the transference number can be increased compared to the pure PE $[39,40]$ while also providing better structural flexibility than an SE [40].

The combination of two different electrolytes does not come without obstacles: charge transfer at the newly created interface between SE and LE can be noticeably hindered and the internal resistance increases. In fact, the activation energy for the LE/SE interface charge transfer was reported to be the highest of all the components in the cell $[5,41]$.

There are quite a few reports about the interface between SEs and LEs, with the earliest ones by Ogumi and co-workers [41-46]. These studies and more recent ones [5, 47-49] almost exclusively rely on electrical (mostly impedance) measurements. Information on the origin of the interface resistances is scarce, and only limited chemical analysis of the interfaces is available. Only recently, a three-dimensional interphase has been reported, comprising decomposition products of the electrolytes [5, 25, 50, 51].

The interface between an SE and a PE is another important subject of research. Compared to the SE/LE interface, the interfacial resistance and the energy barrier for the ion transport between the SE and the PE are mostly both higher [41]. In most of the published work, the interface resistance and activation energy were reported [41, 44, 52-58], whereas the interface chemistry has been investigated only partially so far.

Even less focus was put on the interfacial properties between two different SEs in batteries [8, 35, 36]. Only resistances and activation energy were reported, as well as $\mathrm{X}$-ray diffraction (XRD) data to show the absence of decomposition of the bulk materials $[35,36]$. To gain knowledge about the actual contribution of the interfacial transport between both SEs, a model system is necessary. This could also be used to chemically probe the interfacial region, since chemical degradation and formation of a thin interphase cannot be dismissed [8].

In this review, we summarize reported data on the kinetics of heteroionic interfaces, i.e. interfaces between two different electrolytes, so far. First, data for SE/LE interfaces are presented, which are of major importance for next-generation hybrid $\mathrm{Li}-\mathrm{S}$ and $\mathrm{Li}-\mathrm{O}_{2}$ batteries. Then, we focus on interfaces between inorganic SEs and organic PEs, followed by the combination of two different inorganic SEs. Finally, an overview of the physicochemical models describing these heteroionic interphases will be provided. By summarizing reported data and unsolved problems of heteroionic junctions, we hope to inspire future work on the kinetics of heteroionic interfaces, helping to speed up the development of batteries with hybrid or bilayered electrolytes.

\section{Solid/Liquid Interfaces}

In LIBs, solvated lithium ions transported in the LE from one electrode to the other have to cross two phase boundaries at the cathode/LE and the LE/anode interfaces. At both interfaces, (de)solvation of the lithium ion is necessary. Ogumi and co-workers first explored this important charge-transfer step by temperature-dependent electrochemical impedance spectroscopy (EIS) on model systems using $\mathrm{LiMn}_{2} \mathrm{O}_{4}[59]$ and graphite [60] electrodes in combination with several LEs. They found high activation barriers in both cases and a major influence of the desolvation process.

Later, SEs instead of electrode materials were used as model systems for LIBs to solely observe the interfacial charge transfer without being affected by redox reactions taking place at the electrodes [42]. Four-point EIS measurements eliminate the $\mathrm{Li} / \mathrm{LE}$ interface and only access the bulk and grain boundary resistance of the SE, the resistance of the LE as well as the interfacial resistance of the SE/ LE boundary. Complementary EIS measurements of single components are necessary for these experiments to unambiguously identify all processes. In this first study, the activation energy of the interfacial transfer was independent of the SE, but varied for different LEs. Therefore, the activation barrier depends on the interaction between the lithium ion and the solvent [42]. A trend to higher activation energy for higher Gutmann donor numbers (DNs) of the solvent was found. The DN is a measure of the Lewis basicity. Thus, a higher DN indicates stronger interaction with the Lewis acid $\mathrm{Li}^{+}$[61].

The changes when using $\mathrm{Na}^{+}$ion conductors instead of $\mathrm{Li}^{+}$ion conductors were analyzed as well [44]. In this study, two different sodium-ion conductors were used in combination with sodium trifluoromethanesulfonate (NaTf) in propylene carbonate (PC) or dimethyl sulfoxide (DMSO). Based on the knowledge about $\mathrm{Li}^{+}$transfer across SE/LE interfaces with different solvents [42], DMSO was assumed to result in higher activation energy for the interfacial charge transfer. That is, because of the higher DN of DMSO compared to $\mathrm{PC}$, indicating a higher Lewis basicity and, thus, stronger interaction between the solvent and the sodium ion [61]. The measured activation energy when keeping the same SE was almost the same, though. Therefore, the authors concluded that as long as the chemical potential of $\mathrm{Na}^{+}$in the $\mathrm{SE}$ is lower than that in the LE, the difference between the chemical potential of $\mathrm{Na}^{+}$in the SE and the transition state 
is decisive for the activation barrier. Small differences in the activation energy for different LEs depend, in turn, on the difference in $\mathrm{Na}^{+}$chemical potential between the transition states for different solvents. Finally, the authors suggested that for $\mathrm{Na}^{+}$ions the transfer from the SE to the LE, which is the solvation process, is decisive for the activation energy of the interfacial charge transfer because only a dependence on the SE was found, but not on the LE [44].

Another study compared the interfacial charge transfer of $\mathrm{Li}^{+}$and $\mathrm{Na}^{+}$ions [41]. The activation barriers were higher for $\mathrm{Li}^{+}$than for $\mathrm{Na}^{+}$ions because of the higher Lewis acidity of the $\mathrm{Li}^{+}$ion. A possible increase in the battery rate performance by decreasing the interaction between the solvated lithium ion and the solvent was suggested. Yet, potential detrimental interactions between the LE and the SE were not considered. Instead, the interfacial resistance and the energy barrier of the process were reported to originate only from the desolvation process necessary to allow for the change from diffusion in the LE to a hopping mechanism in the SE (depicted in Fig. 2a).

Studies on binary LEs were conducted to compare different mixing ratios of ethylene carbonate (EC) and dimethyl carbonate (DMC) to pure DMC and evaluate the influence on interface charge transfer [46]. The activation barrier for $\mathrm{Li}^{+}$charge transfer in the binary mixtures was significantly higher. This phenomenon was attributed to the higher solvation ability of EC compared to DMC-the difference of the activation energy in EC/DMC to that in DMC closely resembled the difference in solvation enthalpy of $\mathrm{Li}^{+}$in $\mathrm{EC}$ and $\mathrm{Li}^{+}$in DMC, respectively. However, the activation energy for various mixing ratios of $\mathrm{EC}$ and DMC as almost identical even though the average solvation numbers of EC molecules per $\mathrm{Li}^{+}$vastly differed from each other, as determined via Raman spectroscopy. Therefore, desolvation happens stepwise and the desolvation from the last solvent molecule appears to be rate-determining.

In addition, experiments with different salt concentrations in the same LE were performed [43]. No significant change of the interfacial activation barrier was found when increasing the $\mathrm{LiClO}_{4}$ concentration from $1 \mathrm{~mol} \mathrm{dm}^{-3}$ to $3.95 \mathrm{~mol} \mathrm{dm}^{-3}$. However, at $5.93 \mathrm{~mol} \mathrm{dm}^{-3} \mathrm{LiClO}_{4}$ the activation energy increased by almost $20 \%$. Using Raman spectroscopy, the structure of the coordination shell in the respective LE was studied. At low salt concentration, only solvated $\mathrm{Li}^{+}$ions were detected, while an increasing fraction of solvent-shared ion pairs consisting of $\mathrm{Li}^{+}$and $\mathrm{ClO}_{4}{ }^{-}$was observed with increasing salt concentrations. For $5.93 \mathrm{~mol} \mathrm{dm}^{-3} \mathrm{LiClO}_{4}$, contact ion pairs dominated, explaining the higher activation energy at high concentration. This observation led the authors to the assumption that the desolvation process-as reported previously [46] —is not always the rate-determining step for interfacial charge transport.
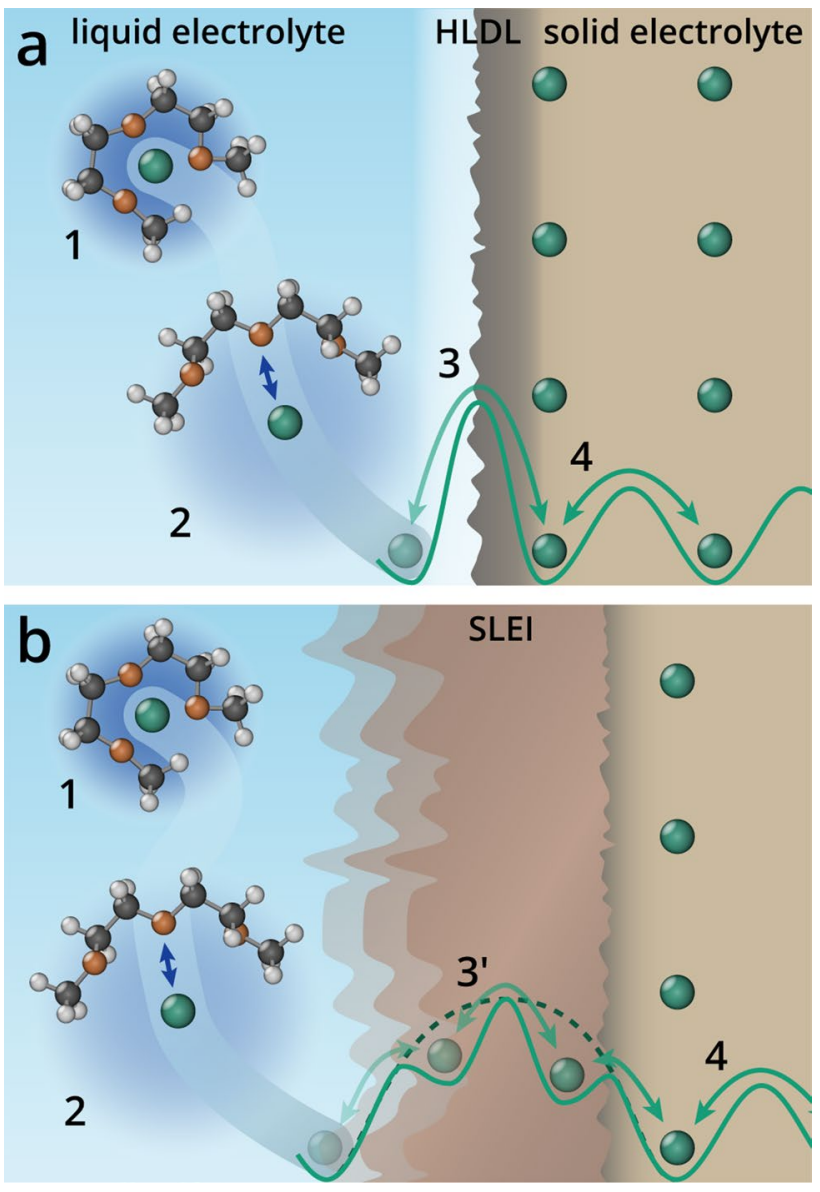

Fig. 2 Schematic representation for the transport of ions across the phase boundary between liquid and solid electrolytes: a established understanding of a charge-transfer resistance at the interface, as reported by Ogumi and co-workers [41-45], and b transport across a resistive three-dimensional solid-liquid electrolyte interphase (SLEI) formed because of decomposition of the electrolytes [5, 25]. The Helmholtz-like double layer (HLDL) at the interface between liquid and solid phases [49] is omitted in (b) for clarity. The transport process involves (1) diffusion of solvated alkali ions (green), (2) desolvation from the last solvent molecule (diglyme is shown in this example), transport across the interface (3) or interphase (3'), and (4) hopping transport in the SE

Instead, they proposed the cleavage of contact ion pairs to be rate-determining in concentrated LEs [43].

To further shed light on the difference between solvation and desolvation in $\mathrm{Li}^{+}$-based systems, a dc voltage was applied during EIS measurements on an asymmetrical setup with one reference electrode in the same compartment with a counter electrode and another reference electrode in a separate compartment [45]. For the utilized cell setup, negative dc voltages cause transport of $\mathrm{Li}^{+}$from the LE to the $\mathrm{SE}$ - the desolvation process - and positive dc voltages drive transport from the SE to the LE- the solvation process. In their experiments, the authors found higher resistances for negative dc voltages when using DMSO as the solvent. 
Therefore, they argued that interfacial $\mathrm{Li}^{+}$transfer is mainly governed by the desolvation process [45].

The results reported up to this point were exclusively obtained via EIS. Another method, only rarely applied, is the $\mathrm{dc}$ polarization technique. First used in a two-electrode setup for the characterization of LE/SE interfaces [47], dc polarization measurements allow for analysis of the resistance depending on the current direction and evaluation of kinetic parameters of the system [49], just like EIS with superimposed dc voltage [45]. Contrary to this earlier EIS study, significant differences between solvation and desolvation resistances in DMSO were not found using dc techniques [47]. However, the measured polarization resistances were in the same order of magnitude as the resistances reported in an earlier work for similar systems [42]. Later, the desolvation/ solvation process was confirmed as the rate-limiting step interfacial $\mathrm{Li}^{+}$transfer via eight-electrode dc polarization measurements [49].

Initially, it was almost generally assumed that the activation barrier for $\mathrm{Li}^{+}$transfer across the SE/LE phase boundary simply originates from the energy necessary for desolvation or solvation of the $\mathrm{Li}^{+}$ion, as depicted in Fig. 2a. According to this assumption, the interfacial charge-transfer process comprises diffusion of the solvated $\mathrm{Li}^{+}$ion to the interface (1), desolvation from the last solvent molecule (2), transfer across the boundary (3), and finally hopping transport in the SE (4). Additionally, the SE/LE system was intentionally selected to describe ion transfer at LE/electrode interfaces because it was assumed that there are no redox reactions and electron transfer steps at the interface between the LE and the SE $[41,43,47]$.

Using only EIS or other electrochemical methods, this was a reasonable assumption since information about the chemistry of the interface is not obtained. However, in a more recent study, a combination of time-dependent EIS and surface analysis with X-ray photoelectron spectroscopy (XPS) and time-of-flight secondary ion mass spectrometry (ToF-SIMS) was utilized on a model system for hybrid $\mathrm{Li}-\mathrm{S}$ batteries consisting of $\mathrm{Li}_{1+x} \mathrm{Al}_{x} \mathrm{Ge}_{2-x}\left(\mathrm{PO}_{4}\right)_{3}$ (LAGP) as the $\mathrm{SE}$ and the ether-based LE [5]. In doing so, a new phase (interphase) that formed at the interface of the LE and the SE upon contact was found. This solid-liquid electrolyte interphase (SLEI) consists of decomposition products of conducting salt, solvents, and the SE. Inorganic ( $\mathrm{LiF}, \mathrm{Li}_{x} \mathrm{SO}_{y}$, carbonates, and phosphates) as well as organic (carbonyl species and alcoholates) and polymeric compounds were found in the SLEI. Also, its resistance was observed to increase over time until reaching a limit.

In a follow-up study, the SLEI formation between different SEs and LEs was analyzed [62]. Its growth on lithium-ion-conducting glass ceramic $\left(\mathrm{Li}_{1+x+y} \mathrm{Al}_{x}(\mathrm{Ti}, \mathrm{Ge})_{2-x} \mathrm{Si}_{y} \mathrm{P}_{3-y} \mathrm{O}_{12}\right.$, LICGC) and lithium phosphorous oxide nitride $\left(\mathrm{Li}_{x} \mathrm{PO}_{y} \mathrm{~N}_{z}\right.$, "LiPON") was confirmed to occur similarly to that on LAGP. Here, lithium bis(trifluoromethanesulfonyl)imide (LiTFSI) was used as the conducting salt in 1,3-dioxolane (DOL), 1,2-dimethoxyethane (DME), and their binary mixture resulted in different growth rates and composition of the formed SLEI. In DME, the SLEI was reported to mainly consist of $\mathrm{LiF}$ and carboxylates while being highly resistive. DOL on the other hand, primarily leads to polymeric species and a lower resistance. For the binary mixture, both phenomena occur, resulting in a mixed SLEI with medium resistance [62].

The occurrence of an SLEI was then confirmed for a conventional $\mathrm{LIB} \mathrm{LE}\left(\mathrm{LiPF}_{6}\right.$ in EC/DMC) and a garnet SE using dc polarization measurements [49]. The authors found a constant ohmic resistance attributed to ionic conduction in the SLEI as well as a term dependent on the salt concentration in the LE. Recently, the same system was analyzed by means of EIS, transmission electron microscopy (TEM), XPS, ToFSIMS, and magic angle spinning nuclear magnetic resonance spectroscopy (MAS NMR) [51]. Decomposition of the SE and the LE resulted in the formation of an SLEI comprising $\mathrm{LiF}, \mathrm{Li}_{2} \mathrm{O}, \mathrm{Li}_{2} \mathrm{CO}_{3}$, and organic fragments. The resistance of the SLEI thereby increased gradually until stablized after about $150 \mathrm{~h}$ [51].

Meanwhile, SLEI formation was also observed on LAGP used as a separator in $\mathrm{Li}-\mathrm{S}$ batteries after cycling [25]. Since the system was closely related to that investigated by Busche et al. [5], similar decomposition products were found. The cell also exhibited remarkable cycling stability because the applied LAGP separator was able to fully suppress the polysulfide shuttle [25].

The interphase formation on "LiPON" thin films immersed in ether-based LEs was investigated using neutron reflectometry (NR), quartz crystal microbalance (QCM) and atomic force microscopy (AFM) measurements [50]. Thereby, SLEI thickness of about $25 \mathrm{~nm}$ was observed after $24 \mathrm{~h}$. The SLEI formation mechanism on "LiPON" was found to involve the quick deposition of a mostly covering interphase with some pinholes left, which are subsequently slowly filled, explaining the increase in the interphase resistance. In these experiments, a two-layer SLEI was observed, with one layer being attributed to inorganic lithium salts and the other to a presumably polymeric material [50].

Considering the formation of such an interphase, the model for ionic transport across the interface has to be modified (Fig. 2b). In addition to two charge-transfer processesthe LE to the SLEI and from the SLEI to the SE-the ionic resistance of the SLEI itself has to be taken into account $\left(3^{\prime}\right)$. Possible transport mechanisms will be discussed in section 5 .

Up to this point, we have considered the mechanistic interpretation of the charge transfer across heteroionic interfaces. In the following, the reported results shall be quantified. The interfacial resistances and activation energy 


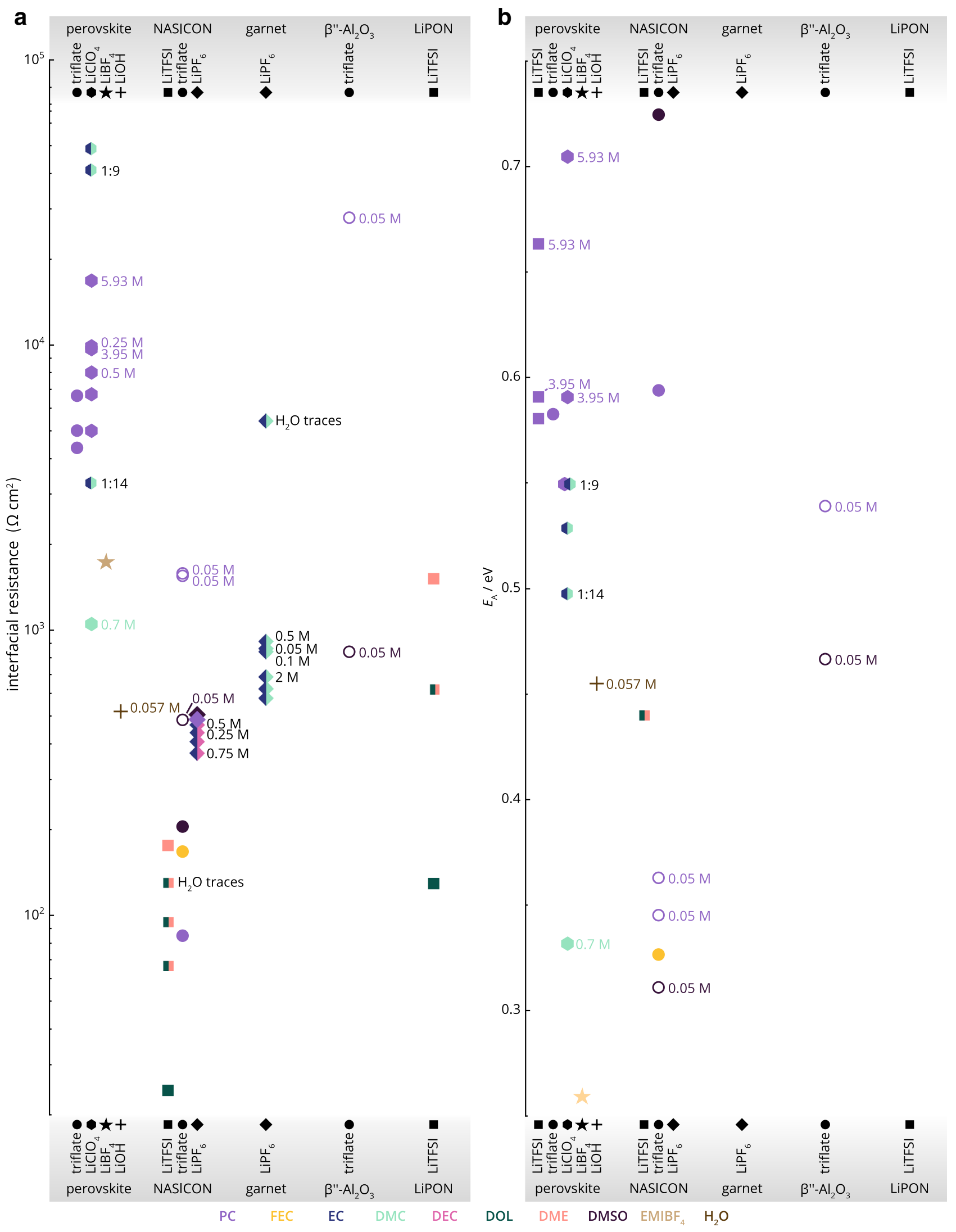


4Fig. 3 Interfacial resistances for various SEs immersed in different LEs at $25^{\circ} \mathrm{C}$ (a) and activation energy for ionic transport across several LE/SE boundaries (b) [5, 41-44, 46-49, 51]. The shape and position of the data points indicate the conducting salt. Its concentration is $1 \mathrm{~mol} \mathrm{dm}^{-3}$ unless specified otherwise. Color indicates the solvent, two-color points represent binary mixtures. The mixture ratio is $1: 1$ unless specified otherwise. Water contamination of the LE is indicated by " $\mathrm{H}_{2} \mathrm{O}$ traces", sodium-ion conduction is depicted by nonfilled data points. The NASICON category contains reported values for LAGP, LICGC, and $\mathrm{Na}_{3} \mathrm{Zr}_{1.88} \mathrm{Y}_{0.12} \mathrm{Si}_{2} \mathrm{PO}_{12}$. All data used for this figure are available in Table $\mathrm{S} 1$ in the Supporting Information

determined in the aforementioned studies are depicted in Fig. 3, the data are summarized in Table S1 in the Supporting Information. Based on this summary, we rank the different combinations of solvents, conducting salts and SEs as follows. Among the SEs, lithium-ion conductors with sodium $(\mathrm{Na})$ super ionic conductor (NASICON) structure like LAGP or LICGC generally result in the lowest interfacial resistance [5, 42, 47]. At higher resistances, one can find LLZO, a garnet-type SE [49], and "LiPON" [62]. The highest interfacial resistances were reported for perovskitetype $\mathrm{Li}_{0.35} \mathrm{La}_{0.55} \mathrm{TiO}_{3}$ (LLTO) [41-43, 46]. The trend that NASICON results in the lowest resistance also holds true for $\mathrm{Na}^{+}$ion conduction. For the identical LE, the interfacial resistance reported was lower than that for sodium betaalumina $\left(\mathrm{Na} \beta^{\prime \prime}-\mathrm{Al}_{2} \mathrm{O}_{3}\right)[41,44]$.

Explaining the correlation between interfacial resistance and the type of SEs is rather difficult, since there are hardly any studies available comparing different SEs. If we consider a stable interface without any decomposition, the activation energy for interfacial charge transfer (and with that the interfacial resistance) depends on the difference of the chemical potential of the alkali ion in the SE and that in the transition state between the SE and the LE. Of these, the transition state is mostly dependent on the LE [44]. Since the chemical potential in the SE is influenced by its crystal structure, the interfacial resistance depends on the SE structure for a stable interface. In real systems, however, decomposition and formation of an SLEI usually occurs at the interface. Then, the interfacial resistance consists of the ionic resistance of the interphase and the charge-transfer resistance for crossing the boundary between the SLEI and the LE (as well as that between the SLEI and the SE), as shown in Fig. 3b. Hence, the structure of the decomposition products also influences the interface resistance. Since only a few studies analyzing the composition of the interphase are available, it is impossible to arrive at a clear conclusion for the dependence between the SE and the resistance. Therefore, these analyses are of great interest for further investigation.

To distinguish between the influence of the conducting salt and that of the solvent is quite challenging, as certain salts are only used with certain solvents and a systematic study is not available. Nevertheless, LiTFSI in ethers results in the lowest resistances [5], whereas lithium trifluoromethanesulfonate (LiTf) in carbonates yields only slightly more resistive interfaces [42]. The resistances measured for $\mathrm{LiPF}_{6}$ in carbonates are even higher [47]. Resistances for $\mathrm{LiClO}_{4}$ in carbonates were only reported in combination with LLTO and a proper trend cannot be observed. The values range from below that found for LiTf in carbonates to over an order of magnitude higher $[41-43,46]$. While the addition of water impurities to organic LEs resulted in higher interphase resistances [5, 49], the lowest resistance reported for LLTO was measured in an aqueous LE [48]. Because of the lack of other studies using aqueous LEs, it is impossible to conclude on a trend.

To analyze the impact of the salt concentration on the $\mathrm{Li}^{+}$ transfer resistance, one has to distinguish between high and low conducting salt concentrations. At low salt concentration, examined in the case of $\mathrm{LiClO}_{4}$ in $\mathrm{PC}$, the interfacial resistance was reported to increase with decreasing salt concentration [42, 43]. On the other hand, an increasing interfacial resistance was observed with increasing salt concentration at high concentration of $\mathrm{LiClO}_{4}$ in PC. The authors explained this with (i) higher viscosity of the LE resulting in worse wettability between the LE and the SE and (ii) a lower degree of dissociation at higher salt concentration leading to a smaller concentration of mobile lithium ions [43].

Activation energy (compare Fig. 3b), on the other hand, strongly depends on the $\mathrm{LE}$ for $\mathrm{Li}^{+}$transport. As described above, the solvents can be ranked according to their DNs, with a higher DN resulting in a higher activation barrier. For the same SE and conducting salt, fluoroethylene carbonate (FEC), thus, gave lower activation energy than PC. DMSO resulted in the highest activation barrier [42, 63]. The lowest activation energy was reported for the ionic liquid 1-ethyl-3-methylimidazolium tetrafluoroborate $\left(\mathrm{EMIBF}_{4}\right)$, which has a lower DN than all the organic solvents [64], confirming the correlation between the DN and activation energy [42].

In addition, the solvation ability of the solvent has to be considered. Even though the DNs of EC and DMC are very similar, the measured activation barrier for DMC is greatly decreased because of its lower solvation ability [46]. At higher salt concentrations, however, the interaction between anions and $\mathrm{Li}^{+}$also plays an important role, leading to higher activation energy at elevated salt concentration [43]. Lastly, for $\mathrm{Na}^{+}$ion transport the interfacial activation energy is lower than that for $\mathrm{Li}^{+}$ion transport and mainly dependent on the SE, as described above $[41,44]$. 


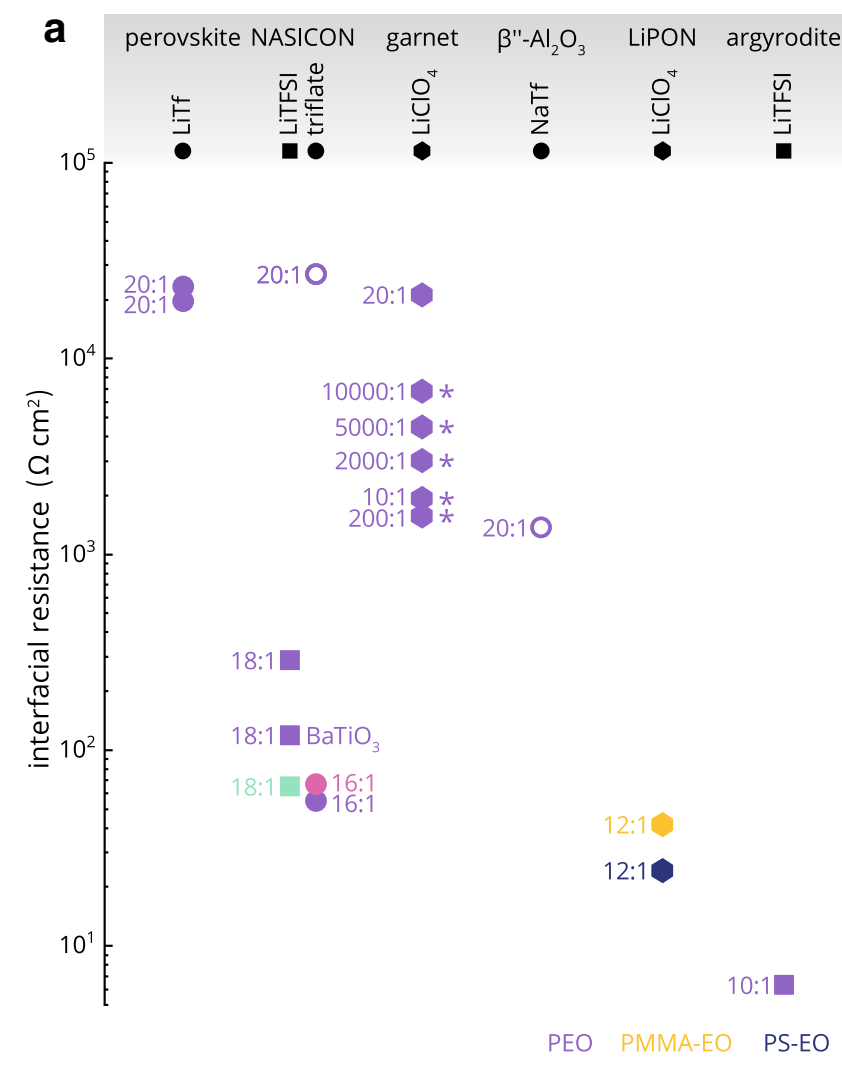

Fig. 4 Interfacial resistances for different SEs in contact with various PEs at $60^{\circ} \mathrm{C}$ (a) and activation energy for ionic transport across various PE/SE interfaces (b) [44, 52-55, 57, 58, 65-67]. The shape and position of the data points indicate the conducting salt. The concentration ratio of the polymer to the conducting salt is given next to the data point. Color indicates the type of polymers used, sodium-ion conduction is depicted by non-filled data points. The NASICON cate-

\section{Solid/Polymer Interfaces}

Compared to the variety of studies available for the LE/ SE interface, only few studies exist for the PE/SE interface. While SEs are used as separators to prevent the chemical cross-talk between the electrodes in LE-systems, PEs are usually applied as interlayers between the lithium metal anode and the SE to prevent interfacial decomposition reactions and improve wettability and contact [28]. Additionally, electrolyte mixtures composed of SE particles dispersed in a PE matrix attracted increasing attention in recent years with the goal of taking advantage of the combined beneficial properties of the PE and the SE. However, the contact between the SE and the PE introduces additional PE/ $\mathrm{SE}$ interfaces contributing to the internal resistance. In order to achieve low overpotentials and long cycle life during operation, a chemically stable interface with low resistance for ion transport is required.

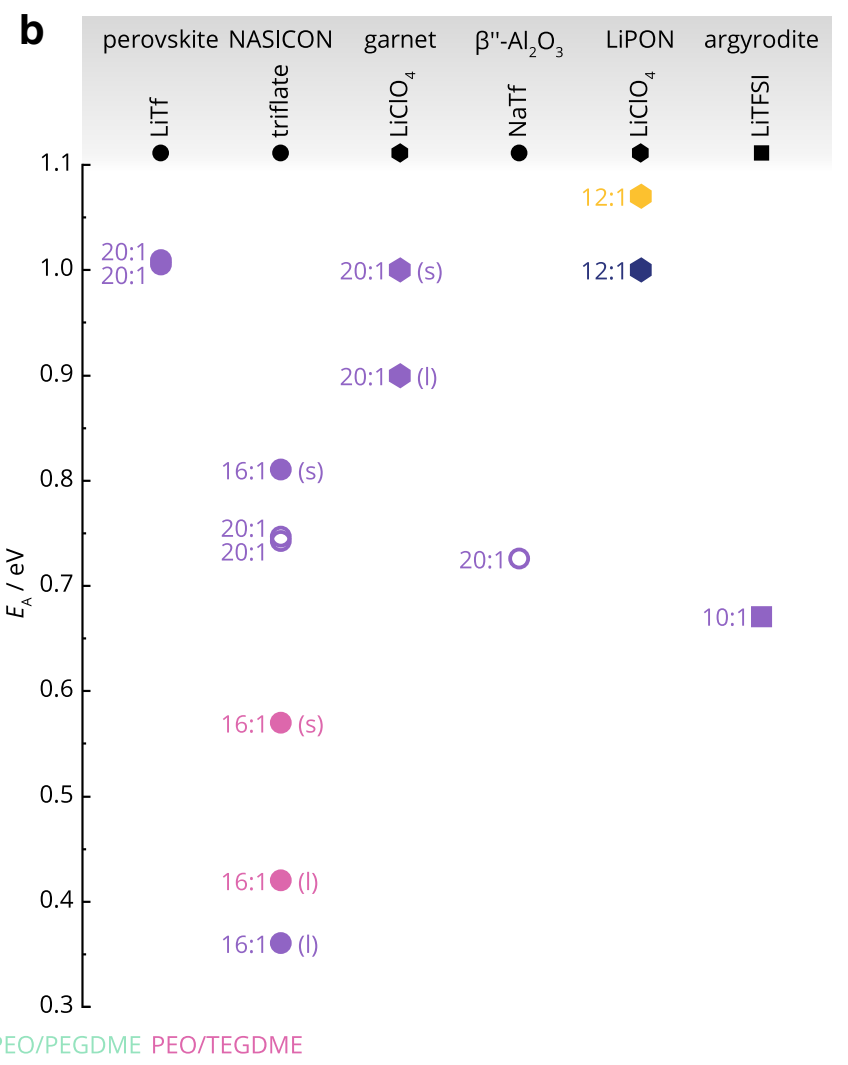

gory contains reported values for LICGC and $\mathrm{Na}_{3} \mathrm{Zr}_{1.88} \mathrm{Y}_{0.12} \mathrm{Si}_{2} \mathrm{PO}_{1} 2$. $\mathrm{BaTiO}_{3}$ was used as the ceramic filler in the PEO matrix. An asterisk indicates measurements at $70^{\circ} \mathrm{C}$. For the activation energy, "(l)" indicates melted PEO, "(s)" solid PEO. All data used for this figure (and additional data at different temperatures) are available in Table S2 in the Supporting Information

Figure 4 summarizes the interface resistance and activation energy of reported PE/SE systems. The studies differ in the parameters chosen: the type of SEs, the polymer and the conducting salt, the salt concentration, the measured temperature range, and the PE fabrication method (slurry- vs. dry-processed).

However, the PE/SE interface resistance used to calculate the activation energy is determined by two-point EIS measurements, while for the LE/SE system usually four-point measurements are used. The two-point setup is prone to errors as the electrode-electrolyte interface is measured in addition to the electrolyte-electrolyte interface (Figure S1 in the Supporting Information).

This complicates drawing general conclusions in terms of favorable ceramics, polymers or salts. The introduction of reference electrodes is a possible way to improve the measurement setup in order to obtain robust data; however, this is rarely done. 
The activation energy of the PE/SE interface in case of lithium-ion conductors is determined around $1 \mathrm{eV}$ in three independent studies with different PE/SE combinations [52, 55, 58]. For example, Abe et al. investigated the interface between LLTO and PEO complexed with LiTf using twopoint EIS measurements [52]. Constant activation energy of $1 \mathrm{eV}$ was obtained for the $\mathrm{Li}^{+}$ion transport through the $\mathrm{PE} / \mathrm{LLTO}$ interface over the measured temperature range $\left((30-90){ }^{\circ} \mathrm{C}\right)$. In contrast, the PE on its own showed a lower, temperature-dependent $E_{\mathrm{A}}$ accounting for $0.53 \mathrm{eV}$ and 0.84 $\mathrm{eV}$ above and below the melting point $\left(60^{\circ} \mathrm{C}\right)$, respectively. Based on the different developments of the activation energy for the interface and the PE bulk, the authors pointed out that the interface structure influences the ion transport.

In a recent study [58], it was shown that a porous interface structure between LLZO and $\mathrm{PEO}_{20}: \mathrm{LiClO}_{4}\left(\mathrm{EO}: \mathrm{Li}^{+}=20: 1\right)$ resulted in a significantly increased resistance while the activation energy was similar to the LLTO/PE discussed above [52]. Fitting the recorded EIS data with a de Levie element-a transmission line model generally used for porous electrodes $[68,69]$ —nabled the distinction between pore and ion transition contributions [58].

The influence of surface impurities on the PE/SE interface resistance has hardly been studied. Regarding the interface between $\mathrm{PEO}_{27}$ :LiTFSI and LLZO:Ta, it was shown that removing (mostly $\mathrm{Li}_{2} \mathrm{CO}_{3}$ ) impurities from the LLZO:Ta surface strongly decreases the interface resistance [70]. However, in order to compare different studies, knowledge of surface properties before contacting the PE and the SE is crucial. Therefore, surface sensitive methods like XPS or ToF-SIMS are useful tools to identify surface species and possibly surface contaminants. Coupling these methods with (four-point) EIS measurements is a good starting point to get a deeper understanding of the occurring (electro)chemistry at the PE/SE interface $[67,71]$. In the case of chemically non-stable PE/SE interfaces, another interesting aspect is the chemical cross-talk between the PE/SE interface and the electrode/electrolyte interface as decomposition products might dissolve in the PE and move to the electrode. So far, only EIS measurements have been reported as an electrochemical characterization method for PE/SE interfaces. However, dc measurements are an interesting alternative to determine the parameters for the reaction kinetics (e.g. exchange current density) as shown for LE/SE interfaces [49]. Additionally, scanning electron microscopy (SEM) is an important technique to identify structure and morphology of the interface [66].

$\mathrm{PE} / \mathrm{SE}$ interface properties are strongly influenced by processing parameters. Keeping the direct contact between the $\mathrm{PE}$ and the SE is essential to mitigate penalties in the ion transport resistance due to contact loss. Therefore, hot-pressing procedures [56] and sputtering techniques [55] proved to be viable methods to decrease the interface resistance compared to stacked SE/PE setups [52, 58].

This was shown for the interface between "LiPON" and poly(styrene-co-poly(ethylene glycol) methyl ether methacrylate) (PS-EO) [56]. Whereas coating the PS-EO electrolyte on top of pre-deposited "LiPON" layers resulted in a significant interface resistance, depositing "LiPON" on top of the PS-EO electrolyte almost eradicated the interface resistance. The corresponding attempt to deposit "LiPON" on top of a poly(methyl methacrylate-co-poly(ethylene glycol)methyl ether methacrylate) (PMMA-EO) copolymer led to the formation of large blisters, impeding further electrochemical characterization [55]. Therefore, we only include the interface resistances and activation energy for the polymer on "LiPON" samples in Fig. 4.

In another approach to form low-resistive PE/SE interfaces, powdered PEO was mixed with LiTFSI or LiTf and directly hot-pressed onto a NASICON-type glass ceramic [56]. The PE/SE resistance contribution showed a minor temperature dependence and was reported as only a fraction of the overall cell resistance. The contact between the $\mathrm{PE}$ and the SE was maintained throughout almost the entire interface of the sample, as evidenced by SEM. This good contact between the layers can be considered as the main reason for the comparably low interface resistance.

Besides the processing parameters, the use of a plasticizer in the PE is another approach to lower the PE/ SE interface resistance. Using a NASICON-type glass ceramic and a spray-coated $\mathrm{PEO}_{16}$ : LiTf, Chen et al. showed that the PE/SE interface resistance can be reduced to virtually zero by adding a small amount of the DMC plasticizer $(\approx 5 \mathrm{wt} \%)$ [66]. Adding DMC was assumed to facilitate the $\mathrm{Li}^{+}$ion transport near the ceramic interface and the $\mathrm{Li}^{+}$ion dissolution from PEO. Additionally, the DMC reduced the activation energy of the PE. The beneficial effect of plasticizers was also shown in a series of studies on aqueous lithium-oxygen batteries, including a $\mathrm{Li}_{1+x} \mathrm{Al}_{x} \mathrm{Ti}_{2-x}\left(\mathrm{PO}_{4}\right)_{3}(\mathrm{LATP}) / \mathrm{PEO}_{18}$ :LiTFSI interface [53, 54, 57]. The incorporation of $\mathrm{BaTiO}_{3}[54]$ or poly(ethylene glycol) dimethyl ether (PEGDME) [57] into the PE membrane positively affected the PE/SE interface resistance. However, as the PE was only applied to protect the LATP against lithium metal, the PE/SE interface was not examined in detail.

In order to elucidate the origin of the SE/PE interface resistance, a combination of two-point EIS measurements and mathematical modeling was carried out for LLZO/PEO: $\mathrm{LiClO}_{4}$ [65]. A strong relation between the salt concentration in the $\mathrm{PE}$ and the interface resistance was observed with a minimum resistance at $\mathrm{Li}^{+}$concentrations between $0.1 \mathrm{~mol} \mathrm{dm}^{-3}$ and $1 \mathrm{~mol} \mathrm{dm}^{-3}$. By considering space-charge effects, the developed model was able to describe the change of the interface resistances over the 
whole $\mathrm{Li}^{+}$-concentration range. While pointing out the importance of space-charge effects in describing the LLZO/ $\mathrm{PE}$ interface, the authors concluded that the activation barrier of the $\mathrm{Li}^{+}$transport between LLZO and the PE is the major contribution to the interface resistance.

In another study, the influence of the LiTFSI salt concentration in PEO on the PEO:LiTFSI/LLZO:Ta interface resistance was elucidated. Varying the EO: $\mathrm{Li}^{+}$salt concentration between $3: 1$ and $27: 1$, the authors showed a minimum interface resistance for the 15:1 ratio. At lower salt concentrations, less lithium ions participate in the lithiumion transport resulting in an increasing interface resistance. However, going to concentrations above 15:1, the authors assumed the precipitation of lithium salt in PEO, also leading to an increasing interface resistance [70].

While most of the published studies use $\mathrm{Li}^{+}$ion conductors, Sagane et al. studied the difference in $\mathrm{Li}^{+}$and $\mathrm{Na}^{+}$transport across the PE/SE and LE/SE interfaces [41]. Therefore, $\mathrm{Li}^{+}$conducting LLTO and $\mathrm{Na}^{+}$conducting NASICON ceramics $\left(\mathrm{Na}_{3} \mathrm{Zr}_{1.88} \mathrm{Y}_{0.12} \mathrm{Si}_{2} \mathrm{PO}_{12}\right)$ in contact with either PEO-based PEs or PC-based LEs were employed as model systems. A significantly higher activation barrier was reported for the PE/SE interface compared to the LE/SE interface. Furthermore, lower activation energy was observed for the $\mathrm{Na}^{+}$-transport through the NASICON/PE interface $(0.74 \mathrm{eV})$ compared to the $\mathrm{Li}^{+}$ion transport through the LLTO/PE interface (1.01 $\mathrm{eV})$. The authors assumed higher solvation energy of $\mathrm{Li}^{+}$ and, hence, a stronger interaction between $\mathrm{Li}^{+}$and the oxygen atoms in PEO. In another study, similar activation energy was observed for the PE/Na $\beta^{\prime \prime}-\mathrm{Al}_{2} \mathrm{O}_{3}$ and the PE/ NASICON interface indicated that the transition state of the transfer process is independent on the type of ceramic electrolytes used [44].

Besides oxide-based SEs, thiophosphate-based SEs attracted increasing attention in the last years. In a recently published work, the interface between $\mathrm{Li}_{10} \mathrm{SnP}_{2} \mathrm{~S}_{12}$ (LSPS) and dry-processed PEO $_{15}$ :LiTFSI was investigated [71]. The formation of polysulfides $\left(\mathrm{S}_{n}^{2-}\right)$, sulfites $\left(\mathrm{SO}_{3}{ }^{2-}\right)$ and $\mathrm{P}-[\mathrm{S}]_{n}-\mathrm{P}$-type bridged $\mathrm{PS}_{4}{ }^{3-}$ units was observed by XPS, ultraviolet-visible spectroscopy (UV-Vis) and ${ }^{31} \mathrm{P}$ MAS NMR. The decomposition reactions were accompanied by an increasing interface resistance measured by two-point EIS. The influence of water impurities and LiTFSI on the LSPS degradation was experimentally excluded, while the influence of the hydroxyl end groups and surface contaminants was shown.

The formation of polysulfides was also observed at the interface between dry-processed $\mathrm{PEO}_{10}$ : LiTFSI and $\mathrm{Li}_{6} \mathrm{PS}_{5} \mathrm{Cl}$; however, additionally the formation of lithium fluoride as a decomposition product of LiTFSI was identified by XPS measurements. Using ToF-SIMS analysis, a thermally induced growth of the formed interphase, which the authors referred to as the solid-polymer electrolyte interphase (SPEI), was shown. Interestingly, the formed SPEI also influenced the lithium/PE interface leading to the formation of a modified solid electrolyte interphase (SEI) with reduced resistance. This observation was explained by polysulfides from the SPEI diffusing through the PE and reacting with the lithium metal electrode. Four-point EIS measurements were carried out, enabling a distinct quantification of the processes occurring at the $\mathrm{PEO}_{10}: \mathrm{LiTFSI} / \mathrm{Li}_{6} \mathrm{PS}_{5} \mathrm{Cl}$ interface. Herein, two resistance contributions were obtained originating from the SPEI $\left(0.3 \Omega \mathrm{cm}^{2}\right.$ at $\left.80^{\circ} \mathrm{C}\right)$ and a charge-transfer process $\left(2.1 \Omega \mathrm{cm}^{2}\right.$ at $\left.80^{\circ} \mathrm{C}\right)$ [67].

Decomposition of thiophosphate SEs was also observed for LPS and $\mathrm{Li}_{10} \mathrm{GeP}_{2} \mathrm{~S}_{12}$ (LGPS) in contact with an etherbased LE [72]. The degree of decomposition was strongly influenced by the LiTFSI salt concentration with a negligible decomposition at high concentrations.

In the "sandwich" systems described so far, relatively well-defined PE/SE interfaces were investigated. In contrast, studies on "mixed" electrolytes composed of SE particles dispersed in a PE matrix usually focus on transport and conduction properties rather than PE/SE interactions. While some publications report the active participation of SE particles in the $\mathrm{Li}^{+}$transport [37], other studies assume that the SE particles act as inactive fillers reducing the PE crystallinity [73]. However, the role of the SE particles in the ion conduction mechanism is still under discussion and a deeper understanding of the PE/SE interface is vital. More comprehensive information on "mixed" electrolytes can be found in a recently published review [8].

\section{Solid/Solid Interfaces}

So far, we have discussed the contact of inorganic SEs with liquids and polymers. With these, wetting of the inorganic electrolyte can be achieved due to the fluidity/plasticity of the liquid or of the polymer at elevated temperature $\left((50-100)^{\circ} \mathrm{C}\right)$. In contrast, using two inorganic SEs causes the challenge of establishing sufficient contact between two hard and brittle materials [8]. To obtain good long-range ionic conductivity in a pellet of an oxide or a phosphate SE, sintering of the powder pellet at high temperature $\left(>500^{\circ} \mathrm{C}\right.$ ) is required. Lithium thiophosphates on the other hand are sufficiently malleable at ambient temperature to achieve high overall ionic conductivity above $1 \mathrm{mS} \mathrm{cm}^{-1}$ through the consolidated phase, which includes grains and grain boundaries.

Grain boundaries are the simplest form of interfaces between two grains of inorganic SEs of the same chemical composition. The conductivity of grain boundaries usually 
limits the overall ionic conductivity of sintered pellets [74]. The brick-layer-model [75] describes the ionic conductivity of such a consolidated phase as high conductivity regions (majority phase) separated from each other by a thin layer of materials of low ionic conductivity. However, not much is known about the exact composition or structure of grain boundaries of SEs. Nonetheless, methods of modifying the grain boundary composition were developed to increase the overall ionic conductivity [76].

The fact that grain boundaries between two grains of the same material can have a strong influence on the transport of $\mathrm{Li}^{+}$brings up the question of how this plays out at the interface between two different inorganic SEs.

In SSBs (as in any battery), a high voltage between a cathode and an anode is desired. Hardly any solid electrolyte is equally suited for reducing and oxidizing conditions at an anode and a cathode [77]. One method to solve this problem is the introduction of coating layers with low electronic conductivity between the SE and the electrode [34]. Although the SE and the coating have to be precisely matched, it is not possible to find the perfect coating material for every SE, however. Another possibility is therefore the application of a consolidated bilayer of two different SEs to separate anode and cathode materials, each suiting the respective active materials. Equally, composites of SE particles embedded in a matrix of a different SE were developed to improve the overall ionic conductivity. Both, the bilayer and the particlein-matrix setups, rely on achieving low interface resistance between the two inorganic SEs.

However, studies have not yet focused on determining the interface resistance between two inorganic SEs. Hence, we draw our conclusions from investigations of SSBs that use two layers of different inorganic SEs, as summarized in Table S3 in the Supporting Information [78-87]. The listed studies all used two thiophosphate-type electrolytes with good ionic conductivity, demonstrating functioning SSBs with either the intercalation-type or the conversiontype cathode active material and either graphite or lithium as the anode active material. The impedance measurements of these cells did not indicate a significant contribution for the transfer of $\mathrm{Li}^{+}$ions between the two SEs. Additionally, many of the presented cells have an overall internal resistance below $100 \Omega \mathrm{cm}^{-2}$ (Table S3 in the Supporting Information). Together, this suggests that the interface resistance between two thiophosphate-type SEs is low, allowing for a good cell performance when using different electrolytes in contact with anodes and cathodes.

With two inorganic SEs, the particle-in-matrix concept was only reported twice, to the best of our knowledge. Both reports embed a garnet-based SE in a thiophosphate-based matrix $[35,36]$. In both studies, the increase in ionic conductivity of $\mathrm{Li}_{3} \mathrm{PS}_{4}$ by the addition of highly conducting LLZO particles suggests that the transfer of lithium ions between thiophosphate to oxide electrolytes is not hindered by a large interface resistance. However, these conclusions still need to be verified by systematic investigations dedicated to determining the interface resistance between a larger number of inorganic SEs.

To the best of our knowledge, the only study directly determining the interfacial resistance between two solid alkali-ion conductors was conducted for the $\mathrm{Na}_{3} \mathrm{Zr}_{2} \mathrm{Si}_{2} \mathrm{PO}_{12} / \mathrm{Na}_{3} \mathrm{PS}_{4}$ interface [88]. An interfacial resistance of $16 \Omega \mathrm{cm}^{2}$ at $25^{\circ} \mathrm{C}$ and activation energy of $0.47 \mathrm{eV}$ were reported, confirming the assumptions drawn above from analyzing the internal resistance of SSBs. The decreased values compared to the $\mathrm{Na}^{+}$transfer at the SE/ $\mathrm{PE}$ interface [41] were explained with a smaller difference in chemical potential of $\mathrm{Na}^{+}$in NASICON and in $\mathrm{Na}_{3} \mathrm{PS}_{4}$ compared to that of $\mathrm{Na}^{+}$in NASICON and the PE [88].

The combination of two different oxide SEs was investigated in the form of LAGP/LATP composite electrolytes [89]. Thereby, the total conductivity could be improved compared to the single LATP or LAGP phases. EIS measurements only showed bulk and grain boundary contributions of the SE, but an interfacial process was not observed. The examined samples were fabricated by mixing and subsequent sintering of both SEs, which led to the formation of a solid solution of the two phases. Therefore, an interface between different SEs was not present [89].

LATP and LAGP were also examined in a bilayer structure [90]. Here, powders of the SEs were pressed on top of each other and subsequently sintered. Like for the LAGP/ LATP composite, an interfacial resistance was not present. This can be explained by the sintering process necessary to achieve sufficient contact between oxide SEs with high elastic modulus. During sintering, interdiffusion of the two materials takes place. Since both SEs were of NASICON structure in the described case [90], degradation is not expected, and only a solid solution is formed. Consequently, the transport path through the bilayer only involves migration through NASICON structures with different concentrations of $\mathrm{Ge}^{4+}$ and $\mathrm{Ti}^{4+}$ ions, which does not exhibit an additional barrier for crossing the interface.

Heterostructural composites of SEs were examined consisting of LATP and LLTO [91]. Sintering of the mixed powders was also necessary for this combination, which led to the formation of dispersed insulating $\mathrm{LaPO}_{4}$ particles and other unidentified decomposition products. Since continuous LATP pathways through the whole pellets were present, an interfacial resistance was not observed. In a bilayer system of LATP and LLTO, however, a high interfacial resistance is to be expected because of the insulating nature of the formed decomposition products. To prove this assumption, further studies are necessary. 


\section{Physical Chemistry of Heteroionic Interfaces}

Basically, the ion transfer across boundaries between two different ionic conductors consists of the sequence as shown in Fig. 2: diffusion to the interface (1), charge transfer across the interface (3), and diffusion from the interface (4). Among these, (1) and (4) can be described by an appropriate model for ion migration, while for (3) an activated jump can be described by Butler-Volmer kinetics, thus a charge-transfer overpotential, can be considered.

Schleutker et al. discussed that the ion transfer across the interface between the LE and the SE can be treated similarly to the electrochemical processes at electrodes [49]. The authors argued that, like the diffusion overpotential at conventional electrodes, the transport of ions to and from the interface can lead to diffusion limitation.

Near the interface, a concentration gradient of mobile ions forms in the Nernst diffusion layer with thickness $\delta_{\mathrm{nl}}$, through which the ions can only move by diffusion. When all the ions reaching the interface are immediately transferred across the boundary, the concentration directly at the interface approaches 0 . The diffusion controlled current density $i_{\mathrm{D}}$ cannot increase further in this case, reaching a maximum

$i_{\mathrm{D}, \max }=z F D \frac{c}{\delta_{\mathrm{nl}}}$

with the charge number $z$, the Faraday constant $F$, the diffusion coefficient $D$, and the concentration $c[49,92,93]$.

Another concept for the interface kinetics between two different (solid) ionic conductors was proposed by Schmalzried and Janek [94]. Assuming that only one type of ions $\left(A^{z+}\right)$ is mobile, the authors analyzed the kinetics of these resting $A X / A Y$ interfaces. In the case of incoherent interfaces (no elastic strain) the interface core is highly disordered, and in the case of (semi-)coherent interfaces the interface region experiences local elastic strain but less disorder. In general, most interfaces considered above are incoherent, and the interface can then be described by an activation barrier due to the disordered core. In addition to the chargetransfer barrier at the interface, the authors also considered deviations from point defect equilibria in the neighboring phases as sources of a (relaxation) overpotential. The authors assumed that the transfer of ions into the neighboring phase initially leads to an excess of ions in non-equilibrium lattice sites. These ions need to relax, which creates a region of defect relaxation, i.e. a kinetically formed layer with different transport properties. In summary, instead of considering a combination of charge transfer and diffusion, the authors considered a combination of charge transfer and point defect relaxation-implicitly assuming fast diffusion [94].
For the actual charge transfer across the boundary, an activated process was also considered by Schleutker et al. [49]. Different chemical potentials of $\mathrm{Li}^{+}\left(\right.$or $\mathrm{Na}^{+}$) ions in the different phases lead to a high difference of the Galvani potential $\Delta \phi_{\mathrm{ct}}$ across the phase boundary. In the case of reorientation of polar solvent molecules and accumulation of oppositely charged ions, the potential difference is localized at the interface region in a Helmholtz-like layer (as shown in Fig. 2) with thickness $d$, which is in the nanometer-range.

In analogy to the Butler-Volmer model for electrode reactions, the current density controlled by thermally activated charge transfer across the phase boundary $i_{\mathrm{ct}}$ can be expressed using the activation energy for transport from the LE to the SE $\Delta G_{\rightarrow}^{\#}$ and $\Delta G_{\leftarrow}^{\#}$ for the transport from solid to liquid as well as a geometry factor $\alpha$ describing the position of the transition state in the Helmholtz-like layer. Furthermore, Schleutker et al. replaced both activation energy by a single one, which they introduced as "reduced" activation energy $\Delta G^{\#}$ using $\alpha$ and the difference of the standard chemical potentials of alkali ions in the liquid and in the solid phases. From that, the exchange current density $i_{0}$ could be calculated using the concentration $c_{A^{z+}, i}$ of ions $A^{z+}$ in the liquid (l) and the solid (s) phases as well as the kinetic rate constants for the charge transfer from liquid to solid $k_{\rightarrow}^{0}$ and from solid to liquid $k_{\leftarrow}^{0}$ according to

$i_{0}=d\left(c_{A^{z+}, 1} k_{\rightarrow}^{0}\right)^{1-\alpha}\left(c_{A^{z+}, \mathrm{s}} k_{\leftarrow}^{0}\right)^{\alpha} \exp \left(-\frac{\Delta G^{\#}}{R T}\right)$

with the gas constant $R$ and the temperature $T$. The polarization resistance $R_{\mathrm{P}, \mathrm{ct}}$ was reported to decrease with increasing concentration of the LE via a power law as

$R_{\mathrm{P}, \mathrm{ct}}=\frac{R T}{z F i_{0}} \propto c_{A X, 1}^{-(1-\alpha)}$,

which was also shown experimentally. However, the authors were only able to fully describe the measured dc polarization curves when accounting for a constant ohmic resistance $R_{\mathrm{SLEI}}$ in series to the current-dependent one, resulting in the total polarization resistance

$R_{\mathrm{P}}=R_{\mathrm{SLEI}}+\frac{R T}{F i_{0}}$.

Thereby, $R_{\mathrm{SLEI}}$ represents the ionic resistance of the formed interphase as shown in Fig. $2 \mathrm{~b}$ and the second term accounts for the charge-transfer resistance across the interface [49].

To decide whether the diffusion to and from the interface or the activated jump across the boundary is ratedetermining, Schmalzried and Janek took the exchange flux of ions $A^{z+}$ across the boundary 
$j_{A^{z+}}^{0}(\mathrm{~b})=\frac{c_{\mathrm{i}}^{0}\left(D_{\mathrm{i}}+D_{\mathrm{V}}\right)^{3 / 2}}{\sqrt{2 \tau_{\mathrm{R}} D_{\mathrm{i}} D_{\mathrm{V}}}}$

with the equilibrium bulk concentration of interstitials $c_{\mathrm{i}}^{0}$, the diffusion coefficients of interstitials $D_{\mathrm{i}}$ and vacancies $D_{\mathrm{V}}$, and the relaxation time $\tau_{\mathrm{R}}$ of the Frenkel reaction into consideration. They concluded that defect relaxation is most probably rate-determining. To corroborate this conclusion, measurements for the exchange flux of $\alpha-\mathrm{AgI} / \beta-\mathrm{Ag}_{2} \mathrm{~S}$ boundaries were presented, leading to relaxation times in the order of those known for Frenkel disorder relaxation $\left(1 \times 10^{-5} \mathrm{~s}\right)$. Therefore, it was concluded that relaxation of point defects at the boundary mainly influences the interfacial resistance [94].

Finally, the formation of space-charge layers at the boundary between two different ionic conductors has to be considered. Because of the different chemical potential of the mobile $A^{z+}$ ions in the neighboring electrolyte phases $A X$ and $A Y$, an electric potential difference is formed. The profile of the electric potential will include a linear profile along the interface core (like a rigid double layer) and a diffuse space-charge layer in both electrolytes. In the case of solid electrolytes with distinct point defects as charge carriers, it can be described in terms of space-charge layers with increased or decreased concentrations of e.g. interstitials and vacancies [95, 96]. Thus, the space-charge layer contains a different concentration of mobile charge carriers (defects), which leads to a different local conductivity and a resistance contribution. In the more typical case of superionic conductors with highly disordered sublattices, depletion and enrichment of ions will also take place, however, with much less local concentration changes and much smaller corresponding local conductivity changes (i.e. also smaller resistance contribution).

Since most SEs are line compounds, even a minuscule compositional change induces an enormous change in the chemical potential [97]. If we now assume that the relation between the chemical potential of ions and the carrier concentration in SEs is similar to that between the chemical potential of the neutral species and the composition, we can also apply the aforementioned consideration to the SE/ SE interface. In this case, balance of the electrochemical potential can be established by tiny changes of the carrier concentration in the interface region. Hence, even if a wide space-charge layer is formed at the interface, the resulting ionic depletion layer-the carrier concentration modulated region-would be very small.

Because of the small decrease of the concentration of mobile charge carriers in the space-charge layer, calculated interface resistances for $\mathrm{Li}^{+}$ion conducting SEs and respective electrode materials were found to be below $1 \Omega \mathrm{cm}^{2}$ [98] and thus too small to explain the measured interfacial resistances for the contact between different lithium-ion conductors. Significantly higher resistances are only expected for complete depletion of $\mathrm{Li}^{+}$ions in the $\mathrm{SE}$, which is highly unlikely for electrostatic reasons. Instead, de Klerk and Wagemaker, who performed the calculations, suggested the formation of decomposition products with low ionic conductivity to be responsible for the experimental values [98], which, again, confirms the reports of SLEI formation as depicted in Fig. $2 b$.

If interstitials $A_{i}^{\cdot}$ and vacancies $\mathrm{V}_{A}^{\prime}$ are formed at the interface, the interfacial resistance should be asymmetric depending on the bias direction. For positive polarization of the $A_{\mathrm{i}}^{*}$ side of the interface and negative polarization of the $\mathrm{V}_{A}^{\prime}$ side, interstitials and vacancies move toward the interface, recombining with each other. Thus, the corresponding interface resistance should be small. For opposite bias on the contrary, defects move away from the depletion layer resulting in higher interfacial resistance. Similar experiments were performed for the LE/SE interface, whereby a different resistance depending on the bias direction was found [45].

In summary, the concept of diffusion overpotential (and the relaxation model as a variant thereof) for transport to and from the interface in combination with charge-transfer analogous to Butler-Volmer kinetics for the actual jump is able to describe the high interfacial resistance between different ionic conductors, while the formation of an ionic depletion layer is not. The fact that Schleutker et al. found a constant ohmic resistance attributed to an SLEI [49] and that the space-charge model is unable to explain the high interfacial resistance [98] suggests that indeed chemical instability of the components forming the interface plays a crucial role. Thus, decomposition products are formed, which often exhibit poor ionic conductivity and therefore lead to a considerably increased total cell resistance. We recommend to check for chemical instability at a heteroionic interface first, before considering depletion layers or chargetransfer kinetics as limiting.

\section{Conclusions}

In this review, the issues originating from ion transfer through different types of heteroionic interfaces were elucidated. Even though the number of reports available on this topic is relatively small, the solid/liquid interface is examined reasonably well. Generally, the charge transfer across the LE/ SE boundary adds a significant resistance to the total cell and is typically accompanied by the highest activation energy of the system. Earlier reports identified the desolvation from the last solvent molecule as the rate-determining step and observed a dependence between activation barrier and Lewis 
basicity of the solvent. Later, it was found that the high interfacial resistance is not only caused by the charge-transfer process but also due to a poorly conducting interphase (SLEI) consisting of decomposition products of the LE and the SE.

For the PE/SE interface, the available data are relatively scarce. Overall, the interphase resistances and activation energy are higher that for for comparable LE systems. Until now, analytical reports on electrolyte decomposition are only available for sulfide-based SEs.

For interfaces between different inorganic solid electrolytes, systematic studies analyzing the interfacial resistance are hardly available. When comparing the internal resistance of SSBs combining two thiophosphate-based SEs, values as low as $14 \Omega \mathrm{cm}^{2}$ were achieved, suggesting lower interfacial resistances than that for SE/LE and SE/PE interfaces.

Finally, an overview of different physicochemical models explaining the interface phenomena on boundaries between distinct ionic conductors was presented leading to the formation of an interphase with poor ionic conductivity as the most probable explanation for high interfacial resistances of heteroionic boundaries.

We anticipate this review to encourage further work on the important topic of electrolyte interface engineering to hopefully develop suitable countermeasures for the significant interface resistances, thus enabling better SSBs in the future.

Acknowledgements Open Access funding provided by Projekt DEAL. The authors acknowledge financial support by BASF SE within the International Network for Electrochemistry and Batteries. They are also grateful for financial support by the Federal Ministry for Education and Research within the FestBatt project (03XP0180).

Open Access This article is licensed under a Creative Commons Attribution 4.0 International License, which permits use, sharing, adaptation, distribution and reproduction in any medium or format, as long as you give appropriate credit to the original author(s) and the source, provide a link to the Creative Commons licence, and indicate if changes were made. The images or other third party material in this article are included in the article's Creative Commons licence, unless indicated otherwise in a credit line to the material. If material is not included in the article's Creative Commons licence and your intended use is not permitted by statutory regulation or exceeds the permitted use, you will need to obtain permission directly from the copyright holder. To view a copy of this licence, visit http://creativecommons.org/licenses/by/4.0/.

\section{References}

1. Tarascon, J.M., Armand, M.: Issues and challenges facing rechargeable lithium batteries. Nature 414, 359-367 (2001). https ://doi.org/10.1038/35104644

2. Goodenough, J.B., Park, K.S.: The Li-ion rechargeable battery: a perspective. J. Am. Chem. Soc. 135, 1167-1176 (2013). https:// doi.org/10.1021/ja3091438

3. Tan, S.J., Zeng, X.X., Ma, Q., et al.: Recent advancements in polymer-based composite electrolytes for rechargeable lithium batteries. Electrochem. Energy Rev. 1, 113-138 (2018). https:// doi.org/10.1007/s41918-018-0011-2
4. Gores, H.J., Barthel, J., Zugmann, S., et al.: Liquid nonaqueous electrolytes. In: Daniel, C., Besenhard, J.O. (eds.) Handbook of Battery Materials, pp. 525-626. Wiley-VCH Verlag GmbH \& Co. KGaA, Weinheim (2011)

5. Busche, M.R., Drossel, T., Leichtweiss, T., et al.: Dynamic formation of a solid-liquid electrolyte interphase and its consequences for hybrid-battery concepts. Nat. Chem. 8, 426-434 (2016). https ://doi.org/10.1038/nchem.2470

6. Janek, J., Zeier, W.G.: A solid future for battery development. Nat. Energy 1, 16141 (2016). https://doi.org/10.1038/nener gy.2016.141

7. Manthiram, A., Yu, X., Wang, S.: Lithium battery chemistries enabled by solid-state electrolytes. Nat. Rev. Mater. 2, 16103 (2017). https://doi.org/10.1038/natrevmats.2016.103

8. Keller, M., Varzi, A., Passerini, S.: Hybrid electrolytes for lithium metal batteries. J. Power Sources 392, 206-225 (2018). https://doi. org/10.1016/j.jpowsour.2018.04.099

9. Mindemark, J., Lacey, M.J., Bowden, T., et al.: Beyond PEOalternative host materials for $\mathrm{Li}^{+}$-conducting solid polymer electrolytes. Prog. Polym. Sci. 81, 114-143 (2018). https://doi. org/10.1016/j.progpolymsci.2017.12.004

10. Maier, J.: Thermodynamics of electrochemical lithium storage. Angew. Chem. Int. Ed. 52, 4998-5026 (2013). https://doi. org/10.1002/anie.201205569

11. Bergner, B.J., Schürmann, A., Peppler, K., et al.: TEMPO: a mobile catalyst for rechargeable $\mathrm{Li}-\mathrm{O}_{2}$ batteries. J. Am. Chem. Soc. 136, 15054-15064 (2014). https://doi.org/10.1021/ja508400m

12. Aurbach, D., McCloskey, B.D., Nazar, L.F., et al.: Advances in understanding mechanisms underpinning lithium-air batteries. Nat. Energy 1, 16128 (2016). https://doi.org/10.1038/nener gy.2016.128

13. Mikhaylik, Y.V., Akridge, J.R.: Polysulfide shuttle study in the Li/S battery system. J. Electrochem. Soc. 151, A1969-A1976 (2004). https://doi.org/10.1149/1.1806394

14. Ji, X., Nazar, L.F.: Advances in Li-S batteries. J. Mater. Chem. 20, 9821-9826 (2010). https://doi.org/10.1039/b925751a

15. Busche, M.R., Adelhelm, P., Sommer, H., et al.: Systematical electrochemical study on the parasitic shuttle-effect in lithiumsulfur-cells at different temperatures and different rates. J. Power Sources 259, 289-299 (2014). https://doi.org/10.1016/j. jpowsour.2014.02.075

16. Sommer, H., Reinacher, J., Janek, J., et al.: Alkali-ion conductive separator assembly for rechargeable electrochemical cells patent US 2016/0141580 A1

17. Imanishi, N., Hasegawa, S., Zhang, T., et al.: Lithium anode for lithium-air secondary batteries. J. Power Sources 185, 1392 1397 (2008). https://doi.org/10.1016/j.jpowsour.2008.07.080

18. Bergner, B.J., Busche, M.R., Pinedo, R., et al.: How to improve capacity and cycling stability for next generation Li- $\mathrm{O}_{2}$ batteries: approach with a solid electrolyte and elevated redox mediator concentrations. ACS Appl. Mater. Interfaces 8, 7756-7765 (2016). https://doi.org/10.1021/acsami.5b10979

19. Kwak, W.J., Jung, H.G., Aurbach, D., et al.: Optimized bicompartment two solution cells for effective and stable operation of Li- $\mathrm{O}_{2}$ batteries. Adv. Energy Mater. 7, 1701232 (2017). https:// doi.org/10.1002/aenm.201701232

20. Wenzel, S., Metelmann, H., Raiß, C., et al.: Thermodynamics and cell chemistry of room temperature sodium/sulfur cells with liquid and liquid/solid electrolyte. J. Power Sources 243, 758765 (2013). https://doi.org/10.1016/j.jpowsour.2013.05.194

21. Wang, Q., Jin, J., Wu, X., et al.: A shuttle effect free lithium sulfur battery based on a hybrid electrolyte. Phys. Chem. Chem. Phys. 16, 21225-21229 (2014). https://doi.org/10.1039/c4cp03694h

22. Wang, Q., Wen, Z., Jin, J., et al.: A gel-ceramic multi-layer electrolyte for long-life lithium sulfur batteries. Chem. Commun. 52, 1637-1640 (2016). https://doi.org/10.1039/c5cc08279j 
23. Wang, C., Yang, Y., Liu, X., et al.: Suppression of lithium dendrite formation by using LAGP-PEO (LiTFSI) composite solid electrolyte and lithium metal anode modified by PEO (LiTFSI) in all-solid-state lithium batteries. ACS Appl. Mater. Interfaces 9, 13694-13702 (2017). https://doi.org/10.1021/acsami.7b00336

24. Yu, X., Bi, Z., Zhao, F., et al.: Hybrid lithium-sulfur batteries with a solid electrolyte membrane and lithium polysulfide catholyte. ACS Appl. Mater. Interfaces 7, 16625-16631 (2015). https://doi. org/10.1021/acsami.5b04209

25. Xu, H., Wang, S., Manthiram, A.: Hybrid lithium-sulfur batteries with an advanced gel cathode and stabilized lithium-metal anode. Adv. Energy Mater. 8, 1800813 (2018). https://doi.org/10.1002/ aenm. 201800813

26. Zhang, T., Zhou, H.: A reversible long-life lithium-air battery in ambient air. Nat. Commun. 4, 1817 (2013). https://doi. org/10.1038/ncomms2855

27. Zhou, W., Wang, S., Li, Y., et al.: Plating a dendrite-free lithium anode with a polymer/ceramic/polymer sandwich electrolyte. J. Am. Chem. Soc. 138, 9385-9388 (2016). https://doi.org/10.1021/ jacs.6b05341

28. Li, Y., Xu, B., Xu, H., et al.: Hybrid polymer/garnet electrolyte with a small interfacial resistance for lithium-ion batteries. Angew. Chem. Int. Ed. 56, 753-756 (2016). https://doi. org/10.1002/anie.201608924

29. Liu, W., Milcarek, R.J., Falkenstein-Smith, R.L., et al.: Interfacial impedance studies of multilayer structured electrolyte fabricated with solvent-casted $\mathrm{PEO}_{10}-\mathrm{LiN}\left(\mathrm{CF}_{3} \mathrm{SO}_{2}\right)_{2}$ and ceramic $\mathrm{Li}_{1.3} \mathrm{Al}_{0.3} \mathrm{Ti}_{1.7}\left(\mathrm{PO}_{4}\right)_{3}$ and its application in all-solid-state lithium ion batteries. J. Electrochem. Energy Convers. Storage 13, 21008 (2016). https://doi.org/10.1115/1.4035294

30. Takada, K., Inada, T., Kajiyama, A., et al.: Solid-state lithium battery with graphite anode. Solid State Ion. 158, 269-274 (2003). https://doi.org/10.1016/s0167-2738(02)00823-8

31. Monroe, C., Newman, J.: The effect of interfacial deformation on electrodeposition kinetics. J. Electrochem. Soc. 151, A880-A886 (2004). https://doi.org/10.1149/1.1710893

32. Monroe, C., Newman, J.: The impact of elastic deformation on deposition kinetics at lithium/polymer interfaces. J. Electrochem. Soc. 152, A396-A404 (2005). https://doi.org/10.1149/1.1850854

33. Barai, P., Higa, K., Srinivasan, V.: Lithium dendrite growth mechanisms in polymer electrolytes and prevention strategies. Phys. Chem. Chem. Phys. 19, 20493-20505 (2017). https://doi. org/10.1039/c7cp03304d

34. Nakamura, T., Amezawa, K., Kulisch, J., et al.: Guidelines for all-solid-state battery design and electrode buffer layers based on chemical potential profile calculation. ACS Appl. Mater. Interfaces 11, 19968-19976 (2019). https://doi.org/10.1021/acsam i.9b03053

35. Rangasamy, E., Sahu, G., Keum, J.K., et al.: A high conductivity oxide-sulfide composite lithium superionic conductor. J. Mater. Chem. A 2, 4111-4116 (2014). https://doi.org/10.1039/c3ta1 $5223 \mathrm{e}$

36. Hood, Z.D., Wang, H., Li, Y., et al.: The "filler effect": a study of solid oxide fillers with $\beta$ - $\mathrm{Li}_{3} \mathrm{PS}_{4}$ for lithium conducting electrolytes. Solid State Ion. 283, 75-80 (2015). https://doi.org/10.1016/j. ssi.2015.10.014

37. Zheng, J., Tang, M., Hu, Y.Y.: Lithium ion pathway within $\mathrm{Li}_{7} \mathrm{La}_{3} \mathrm{Zr}_{2} \mathrm{O}_{12}$-polyethylene oxide composite electrolytes. Angew. Chem. 128, 12726-12730 (2016). https://doi.org/10.1002/ ange. 201607539

38. Judez, X., Zhang, H., Li, C., et al.: Polymer-rich composite electrolytes for all-solid-state Li-S cells. J. Phys. Chem. Lett. 8, 3473-3477 (2017). https://doi.org/10.1021/acs.jpclett.7b01321

39. Ban, X., Zhang, W., Chen, N., et al.: A high-performance and durable poly(ethylene oxide)-based composite solid electrolyte for all solid-state lithium battery. J. Phys. Chem. C 122, 9852-9858 (2018). https://doi.org/10.1021/acs.jpcc.8b02556

40. Xie, H., Yang, C., Fu, K.K., et al.: Flexible, scalable, and highly conductive garnet-polymer solid electrolyte templated by bacterial cellulose. Adv. Energy Mater. 8, 1703474 (2018). https://doi. org/10.1002/aenm.201703474

41. Sagane, F., Abe, T., Iriyama, Y., et al.: $\mathrm{Li}^{+}$and $\mathrm{Na}^{+}$transfer through interfaces between inorganic solid electrolytes and polymer or liquid electrolytes. J. Power Sources 146, 749-752 (2005). https://doi.org/10.1016/j.jpowsour.2005.03.075

42. Abe, T., Sagane, F., Ohtsuka, M., et al.: Lithium-ion transfer at the interface between lithium-ion conductive ceramic electrolyte and liquid electrolyte-A key to enhancing the rate capability of lithium-ion batteries. J. Electrochem. Soc. 152, A2151-A2154 (2005). https://doi.org/10.1149/1.2042907

43. Sagane, F., Abe, T., Ogumi, Z.: Li ${ }^{+}$-ion transfer through the interface between $\mathrm{Li}^{+}$-ion conductive ceramic electrolyte and $\mathrm{Li}^{+}$-ionconcentrated propylene carbonate solution. J. Phys. Chem. C 113, 20135-20138 (2009). https://doi.org/10.1021/jp908623c

44. Sagane, F., Abe, T., Ogumi, Z.: Sodium-ion transfer at the interface between ceramic and organic electrolytes. J. Power Sources 195, 7466-7470 (2010). https://doi.org/10.1016/j.jpows our.2010.04.054

45. Sagane, F., Miyazaki, K., Fukutsuka, T., et al.: Lithium-ion transfer at the interface between solid and liquid electrolytes under applying DC voltage. Chem. Lett. 39, 826-827 (2010). https:// doi.org/10.1246/cl.2010.826

46. Yamada, Y., Sagane, F., Iriyama, Y., et al.: Kinetics of lithiumion transfer at the interface between $\mathrm{Li}_{0.35} \mathrm{La}_{0.55} \mathrm{TiO}_{3}$ and binary electrolytes. J. Phys. Chem. C 113, 14528-14532 (2009). https:// doi.org/10.1021/jp9043539

47. Mehrotra, A., Ross, P.N., Srinivasan, V.: Quantifying polarization losses in an organic liquid electrolyte/single ion conductor interface. J. Electrochem. Soc. 161, A1681-A1690 (2014). https ://doi.org/10.1149/2.0721410jes

48. Uhlmann, C., Braun, P., Illig, J., et al.: Interface and grain boundary resistance of a lithium lanthanum titanate $\left(\mathrm{Li}_{3 x} \mathrm{La}_{2 / 3-x} \mathrm{TiO}_{3}\right.$, LLTO) solid electrolyte. J. Power Sources 307, 578-586 (2016). https://doi.org/10.1016/j.jpowsour.2016.01.002

49. Schleutker, M., Bahner, J., Tsai, C.L., et al.: On the interfacial charge transfer between solid and liquid $\mathrm{Li}^{+}$electrolytes. Phys. Chem. Chem. Phys. 19, 26596-26605 (2017). https://doi. org/10.1039/c7cp05213h

50. Weiss, M., Seidlhofer, B.K., Geiß, M., et al.: Unraveling the formation mechanism of solid-liquid electrolyte interphases on LiPON thin films. ACS Appl. Mater. Interfaces 11, 9539-9547 (2019). https://doi.org/10.1021/acsami.8b19973

51. Liu, J., Gao, X., Hartley, G.O., et al.: The interface between $\mathrm{Li}_{6.5} \mathrm{La}_{3} \mathrm{Zr}_{1.5} \mathrm{Ta}_{0.5} \mathrm{O}_{12}$ and liquid electrolyte. Joule (2019). https ://doi.org/10.1016/j.joule.2019.10.001

52. Abe, T., Ohtsuka, M., Sagane, F., et al.: Lithium ion transfer at the interface between lithium-ion-conductive solid crystalline electrolyte and polymer electrolyte. J. Electrochem. Soc. 151, A1950-A1953 (2004). https://doi.org/10.1149/1.1804813

53. Zhang, T., Imanishi, N., Hasegawa, S., et al.: Li/polymer electrolyte/water stable lithium-conducting glass ceramics composite for lithium-air secondary batteries with an aqueous electrolyte. J. Electrochem. Soc. 155, A965-A969 (2008). https://doi. org/10.1149/1.2990717

54. Zhang, T., Imanishi, N., Hasegawa, S., et al.: Water-stable lithium anode with the three-layer construction for aqueous lithium-air secondary batteries. Electrochem. Solid-State Lett. 12, A132A135 (2009). https://doi.org/10.1149/1.3125285

55. Tenhaeff, W.E., Yu, X., Hong, K., et al.: Ionic transport across interfaces of solid glass and polymer electrolytes for lithium ion 
batteries. J. Electrochem. Soc. 158, A1143-A1149 (2011). https ://doi.org/10.1149/1.3625281

56. Tenhaeff, W.E., Perry, K.A., Dudney, N.J.: Impedance characterization of $\mathrm{Li}$ ion transport at the interface between laminated ceramic and polymeric electrolytes. J. Electrochem. Soc. 159, A2118-A2123 (2012). https://doi.org/10.1149/2.063212jes

57. Wang, H., Im, D., Lee, D.J., et al.: A composite polymer electrolyte protect layer between lithium and water stable ceramics for aqueous lithium-air batteries. J. Electrochem. Soc. 160, A728A733 (2013). https://doi.org/10.1149/2.020306jes

58. Langer, F., Palagonia, M.S., Bardenhagen, I., et al.: Impedance spectroscopy analysis of the lithium ion transport through the $\mathrm{Li}_{7} \mathrm{La}_{3} \mathrm{Zr}_{2} \mathrm{O}_{12} / \mathrm{P}(\mathrm{EO})_{20} \mathrm{Li}$ interface. J. Electrochem. Soc. 164, A2298-A2303 (2017). https://doi.org/10.1149/2.0381712jes

59. Yamada, I., Abe, T., Iriyama, Y., et al.: Lithium-ion transfer at $\mathrm{LiMn}_{2} \mathrm{O}_{4}$ thin film electrode prepared by pulsed laser deposition. Electrochem. Commun. 5, 502-505 (2003). https://doi. org/10.1016/S1388-2481(03)00113-9

60. Abe, T., Fukuda, H., Iriyama, Y., et al.: Solvated Li-ion transfer at interface between graphite and electrolyte. J. Electrochem. Soc. 151, A1120-A1123 (2004). https://doi.org/10.1149/1.1763141

61. Gutmann, V.: Solvent effects on the reactivities of organometallic compounds. Coord. Chem. Rev. 18, 225-255 (1976). https://doi. org/10.1016/S0010-8545(00)82045-7

62. Busche, M.R., Leichtweiss, T., Fiedler, C., et al.: The formation of the solid-/liquid electrolyte interphase (SLEI) on NASICON-type glass ceramics and LiPON (2020). https://doi.org/10.26434/chemr xiv. $11734740 . v 1$

63. Nambu, N., Takahashi, R., Takehara, M., et al.: Electrolytic characteristics of fluoroethylene carbonate for electric double-layer capacitors at high concentrations of electrolyte. Electrochemistry 81, 817-819 (2013). https://doi.org/10.5796/electrochemistr y. 81.817

64. Schmeisser, M., Illner, P., Puchta, R., et al.: Gutmann donor and acceptor numbers for ionic liquids. Chem. Eur. J. 18, 1096910982 (2012). https://doi.org/10.1002/chem.201200584

65. Brogioli, D., Langer, F., Kun, R., et al.: Space-charge effects at the $\mathrm{Li}_{7} \mathrm{La}_{3} \mathrm{Zr}_{2} \mathrm{O}_{12} /$ poly (ethylene oxide) interface. ACS Appl. Mater. Interfaces 11, 11999-12007 (2019). https://doi.org/10.1021/acsam i. 8 b 19237

66. Chen, X.C., Liu, X., Pandian, A.S., et al.: Determining and minimizing resistance for ion transport at the polymer/ceramic electrolyte interface. ACS Energy Lett. 4, 1080-1085 (2019). https:// doi.org/10.1021/acsenergylett.9b00495

67. Simon, F.J., Hanauer, M., Henss, A., et al.: Properties of the interphase formed between argyrodite-type $\mathrm{Li}_{6} \mathrm{PS}_{5} \mathrm{Cl}$ and polymerbased PEO $_{10}$ :LiTFSI. ACS Appl. Mater. Interfaces 11, 4218642196 (2019). https://doi.org/10.1021/acsami.9b14506

68. de Levie, R.: Electrochemical response of porous and rough electrodes. Adv. Electrochem. Electrochem. Eng. 6, 329-397 (1967)

69. Barcia, O.E., Elia, E., Frateur, I., et al.: Application of the impedance model of de Levie for the characterization of porous electrodes. Electrochim. Acta 47, 2109-2116 (2002). https://doi. org/10.1016/s0013-4686(02)00081-6

70. Gupta, A., Sakamoto, J.: Controlling ionic transport through the PEO-LiTFSI/LLZTO interface. Electrochem. Soc. Interface 28, 63-69 (2019). https://doi.org/10.1149/2.f06192if

71. Riphaus, N., Stiaszny, B., Beyer, H., et al.: Understanding chemical stability issues between different solid electrolytes in all-solidstate batteries. J. Electrochem. Soc. 166, A975-A983 (2019). https://doi.org/10.1149/2.0351906jes

72. Oh, D.Y., Nam, Y.J., Park, K.H., et al.: Excellent compatibility of solvate ionic liquids with sulfide solid electrolytes: toward favorable ionic contacts in bulk-type all-solid-state lithium-ion batteries. Adv. Energy Mater. 5, 1500865 (2015). https://doi.org/10.1002/ aenm. 201500865
73. Tao, X., Liu, Y., Liu, W., et al.: Solid-state lithium-sulfur batteries operated at $37{ }^{\circ} \mathrm{C}$ with composites of nanostructured $\mathrm{Li}_{7} \mathrm{La}_{3} \mathrm{Zr}_{2} \mathrm{O}_{12}$ /carbon foam and polymer. Nano Lett. 17, $2967-$ 2972 (2017). https://doi.org/10.1021/acs.nanolett.7b00221

74. Xu, R.C., Xia, X.H., Zhang, S.Z., et al.: Interfacial challenges and progress for inorganic all-solid-state lithium batteries. Electrochim. Acta 284, 177-187 (2018). https://doi.org/10.1016/j.elect acta.2018.07.191

75. Bauerle, J.E.: Study of solid electrolyte polarization by a complex admittance method. J. Phys. Chem. Solids 30, 2657-2670 (1969). https://doi.org/10.1016/0022-3697(69)90039-0

76. Lee, S.D., Jung, K.N., Kim, H., et al.: Composite electrolyte for all-solid-state lithium batteries: low-temperature fabrication and conductivity enhancement. ChemSusChem 10, 2175-2181 (2017). https://doi.org/10.1002/cssc.201700104

77. Zhu, Y., He, X., Mo, Y.: Origin of outstanding stability in the lithium solid electrolyte materials: insights from thermodynamic analyses based on first-principles calculations. ACS Appl. Mater. Interfaces 7, 23685-23693 (2015). https://doi.org/10.1021/acsam i. 5 b0 07517

78. Woo, J.H., Trevey, J.E., Cavanagh, A.S., et al.: Nanoscale interface modification of $\mathrm{LiCoO}_{2}$ by $\mathrm{Al}_{2} \mathrm{O}_{3}$ atomic layer deposition for solid-state Li batteries. J. Electrochem. Soc. 159, A1120-A1124 (2012). https://doi.org/10.1149/2.085207jes

79. Xie, D., Chen, S., Zhang, Z., et al.: $\mathrm{Sb}_{2} \mathrm{O}_{5}$-doped $\mathrm{Li}_{3} \mathrm{PS}_{4}$ with excellent stability against $\mathrm{Li}$ for all-solid-state lithium batteries. J. Power Sources 389, 140-147 (2018). https://doi.org/10.1016/j. jpowsour.2018.04.021

80. Kato, Y., Hori, S., Saito, T., et al.: High-power all-solid-state batteries using sulfide superionic conductors. Nat. Energy (2016). https://doi.org/10.1038/nenergy.2016.30

81. Kato, Y., Shiotani, S., Morita, K., et al.: All-solid-state batteries with thick electrode configurations. J. Phys. Chem. Lett. 9, 607-613 (2018). https://doi.org/10.1021/acs.jpclett.7b02880

82. Yao, X., Liu, D., Wang, C., et al.: High-energy all-solid-state lithium batteries with ultralong cycle life. Nano Lett. 16, 71487154 (2016). https://doi.org/10.1021/acs.nanolett.6b03448

83. Zhang, Q., Peng, G., Mwizerwa, J.P., et al.: Nickel sulfide anchored carbon nanotubes for all-solid-state lithium batteries with enhanced rate capability and cycling stability. J. Mater. Chem. A 6, 12098-12105 (2018). https://doi.org/10.1039/c8ta0 $3449 \mathrm{~d}$

84. Shin, B.R., Nam, Y.J., Oh, D.Y., et al.: Comparative study of $\mathrm{TiS}_{2} / \mathrm{Li}$-in all-solid-state lithium batteries using glass-ceramic $\mathrm{Li}_{3} \mathrm{PS}_{4}$ and $\mathrm{Li}_{10} \mathrm{GeP}_{2} \mathrm{~S}_{12}$ solid electrolytes. Electrochim. Acta 146, 395-402 (2014). https://doi.org/10.1016/j.elect acta.2014.08.139

85. Zhang, Q., Mwizerwa, J.P., Wan, H., et al.: $\mathrm{Fe}_{3} \mathrm{~S}_{4} @ \mathrm{Li}_{7} \mathrm{P}_{3} \mathrm{~S}_{11}$ nanocomposites as cathode materials for all-solid-state lithium batteries with improved energy density and low cost. J. Mater. Chem. A 5, 23919-23925 (2017). https://doi.org/10.1039/c7ta0 $7972 \mathrm{a}$

86. Park, K.H., Oh, D.Y., Choi, Y.E., et al.: Solution-processable glass $\mathrm{LiI}_{-} \mathrm{Li}_{4} \mathrm{SnS}_{4}$ superionic conductors for all-solid-state Liion batteries. Adv. Mater. 28, 1874-1883 (2015). https://doi. org/10.1002/adma.201505008

87. Suyama, M., Kato, A., Sakuda, A., et al.: Lithium dissolution/ deposition behavior with $\mathrm{Li}_{3} \mathrm{PS}_{4}$ - $\mathrm{LiI}$ electrolyte for all-solidstate batteries operating at high temperatures. Electrochim. Acta 286, 158-162 (2018). https://doi.org/10.1016/j.elect acta.2018.07.227

88. Noi, K., Nagata, Y., Hakari, T., et al.: Oxide-based composite electrolytes using $\mathrm{Na}_{3} \mathrm{Zr}_{2} \mathrm{Si}_{2} \mathrm{PO}_{12} / \mathrm{Na}_{3} \mathrm{PS}_{4}$ interfacial ion transfer. ACS Appl. Mater. Interfaces 10, 19605-19614 (2018). https:// doi.org/10.1021/acsami.8b02427 
89. Ling, S.-G., Peng, J.-Y., Yang, Q., et al.: Enhanced ionic conductivity in LAGP/LATP composite electrolyte. Chin. Phys. B 27, 38201 (2018). https://doi.org/10.1088/1674-1056/27/3/038201

90. Zhao, E., Ma, F., Guo, Y., et al.: Stable LATP/LAGP doublelayer solid electrolyte prepared via a simple dry-pressing method for solid state lithium ion batteries. R. Soc. Chem. Adv. 6, 92579-92585 (2016). https://doi.org/10.1039/C6RA19415J

91. Onishi, H., Takai, S., Yabutsuka, T., et al.: Synthesis and electrochemical properties of LATP-LLTO lithium ion conductive composites. Electrochemistry 84, 967-970 (2016). https://doi. org/10.5796/electrochemistry.84.967

92. Hamann, C.H., Vielstich, W.: Elektrochemie, 4th edn. Wiley$\mathrm{VCH}$, Weinheim (2005)

93. Wedler, G.: Lehrbuch der physikalischen Chemie, 5th edn. Wiley-VCH, Weinheim (2010)

94. Schmalzried, H., Janek, J.: Chemical kinetics of phase boundaries in solids. Ber. Bunsenges. Phys. Chem. 102, 127-143 (1998). https://doi.org/10.1002/bbpc.19981020202

95. Maier, J.: Space charge regions in solid two phase systems and their conduction contribution-II contact equilibrium at the interface of two ionic conductors and the related conductivity effect. Ber. Bunsenges. Phys. Chem. 89, 355-362 (1985). https ://doi.org/10.1002/bbpc.19850890402

96. Maier, J.: Ionic conduction in space charge regions. Prog. Solid State Chem. 23, 171-263 (1995). https://doi.org/10.1016/00796786(95)00004-e

97. DeHoff, R.: Thermodynamics in Materials Science, 2nd edn. CRC Press, Hoboken (2006)

98. de Klerk, N.J.J., Wagemaker, M.: Space-charge layers in allsolid-state batteries; important or negligible? ACS Appl. Energy Mater 10, 5609-5618 (2018). https://doi.org/10.1021/acsae m. $8 \mathrm{~b} 01141$

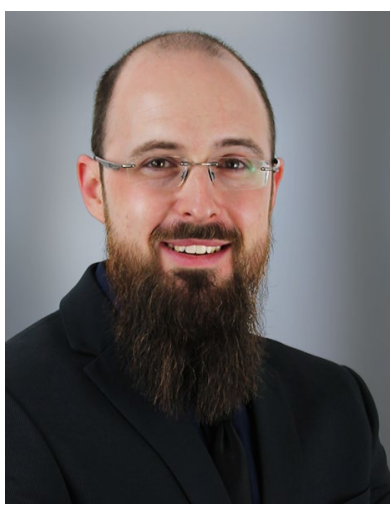

Manuel Weiss received his M.Sc. in Advanced Materials from Justus Liebig University Giessen. Currently, he is working as a $\mathrm{Ph} . \mathrm{D}$. student under supervision of Prof. Jürgen Janek. His research interests include interfacial processes between different ionic conductors as well as structure-property relationships in solid electrolytes.

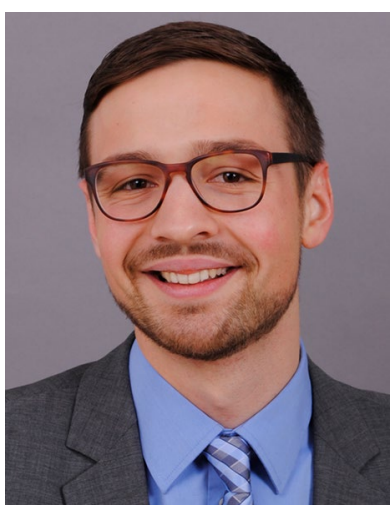

Fabian J. Simon obtained his M.Sc. in Chemistry at Technical University of Munich, Germany, in 2017. He is currently a Ph.D. student working in Professor Janek's group at Justus Liebig University Giessen, Germany. His research focuses on interfaces between solid polymer electrolytes and thiophosphatebased solid electrolytes.

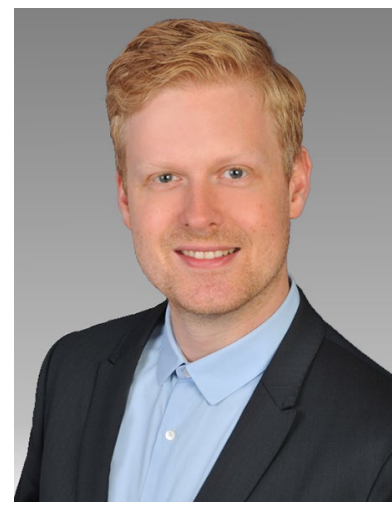

Martin R. Busche studied physics and chemistry at the Justus Liebig University Giessen and received his $\mathrm{Ph} . \mathrm{D}$. in the field of physical chemistry under the supervision of Prof. Dr. Jürgen Janek. His research mainly focuses on new generation battery systems, especially featuring hybrid cell concepts comprising liquid and solid electrolytes. Herein, the novel phenomenon of a solid-liquid electrolyte interphase (SLEI) evolving at the heterogeneous phase boundary was found and analyzed in 2014

He worked for the Robert Bosch GmbH in multiple HV and 48V projects. Currently, he is Head of Engineering in the Farasis Energy Europe $\mathrm{GmbH}$, the European entity of a global supplier of Li-Ion Battery Systems for automotive, stationary and industrial applications.

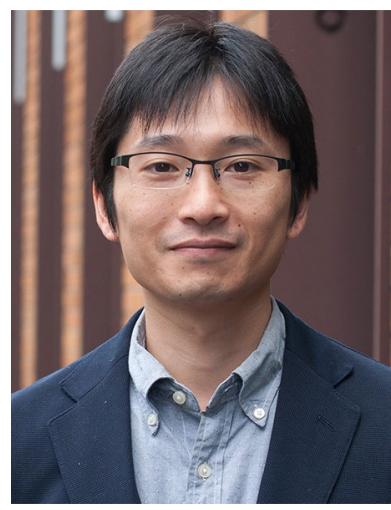

Takashi Nakamura received his Ph.D. from Tohoku University in 2010. After working at Sumitomo Metal Mining Co., Ltd. as a researcher, he joined Institute of Multidisciplinary Research for Advanced Materials at Tohoku University in 2012 as an Assistant Professor, and currently, he is an Associate Professor at the Tohoku University. His research interests are development of energy storage and conversion technologies such as lithium-ion batteries, all-solidstate batteries and solid oxide fuel cells based on solid-state electrochemistry and defect chemistry.

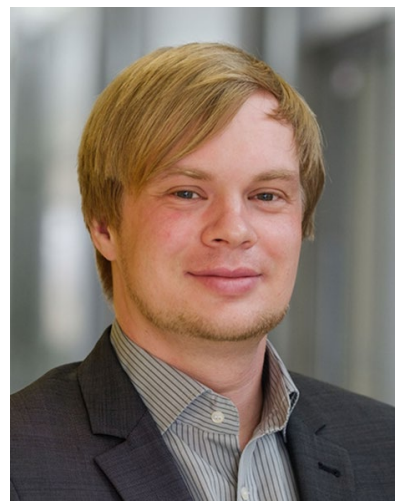

Daniel Schröder received his $\mathrm{Ph} . \mathrm{D}$. in process engineering with distinction from TU Braunschweig in 2015 , researching on secondary zinc-oxygen batteries. Afterward, he joined the Institute of Physical Chemistry at Justus Liebig University Giessen as a junior group leader. His research group focuses on redox-flow batteries and metal-oxygen batteries 
as well as on the understanding of the interfaces between liquid and solid in those batteries.

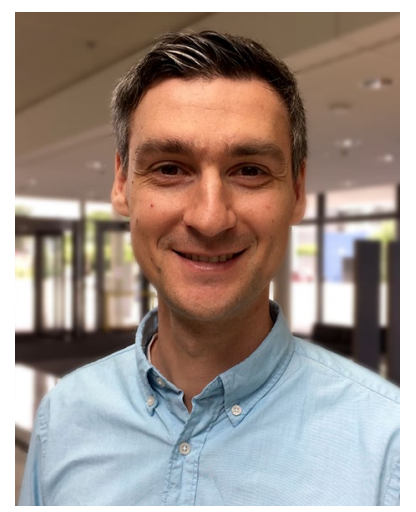

Felix H. Richter received his Ph.D. in chemistry with distinction from Ruhr-Universität Bochum in 2013 for his work on mesoporous polymers as catalysts for the conversion of biomass, which he carried out at the Max-Planck-Institut für Kohlenforschung in Mülheim an der Ruhr. During his postdoctoral work at the Department of Chemistry, University of St Andrews, he obtained a Research Fellowship from the Deutsche Forschungsgemeinschaft to work on bicontinuous hybrid electrolytes for solid-state batteries at the Department of Materials, University of Oxford. He then joined the Institute of Physical Chemistry at Justus Liebig University Giessen where he became a junior group leader. His research focuses on developing hybrid electrolytes for solid-state batteries and on understanding ion transport within composite solid electrolytes.

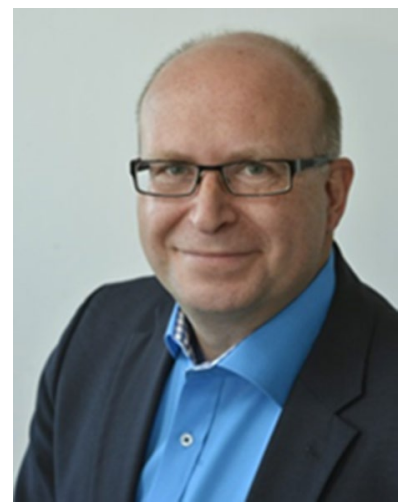

Jürgen Janek holds a chair for Physical Chemistry at Justus Liebig University in Giessen (Germany) and is scientific director of BELLA, a joint lab of BASF SE and KIT in Karlsruhe/ Germany. He received his doctoral degree in physical chemistry and was visiting professor at Seoul National University, Tohoku University and Université d'Aix-Marseille. His research spans a wide range from transport studies in mixed conductors and at interfaces to in situ studies in electrochemical cells. Current key interests include all-solid-state batteries, solid electrolytes and solid electrolyte interfaces. He is particularly interested in kinetics at interfaces. 